\title{
ON THE THOM-BOARDMAN SYMBOLS FOR POLYNOMIAL MULTIPLICATION MAPS*
}

\author{
JIAYUAN LIN ${ }^{\dagger}$ AND JANICE WETHINGTON ${ }^{\ddagger}$
}

\begin{abstract}
The Thom-Boardman symbol was first introduced by Thom in 1956 to classify singularities of differentiable maps. It was later generalized by Boardman to a more general setting. Although the Thom-Boardman symbol is realized by a sequence of non-increasing, nonnegative integers, to compute those numbers is, in general, extremely difficult. In the case of polynomial multiplication maps, Robert Varley conjectured that computing the Thom-Boardman symbol for polynomial multiplication reduces to computing the successive quotients and remainders for the Euclidean algorithm applied to the degrees of the two polynomials. In this paper, we confirm Varley's conjecture.
\end{abstract}

Key words. Thom-Boardman symbols, polynomial multiplication maps, Toeplitz matrices.

AMS subject classifications. 58K40, 58K20, 32S10, 14J17.

1. Introduction. This paper proves Robert Varley's conjecture on the ThomBoardman symbols for polynomial multiplication maps.

In 1956, R. Thom developed a method to classify singularities of differentiable maps according to the rank of the first differential of the map and the ranks of its restrictions to submanifolds of singularities. His theory depended upon the manifold structure of the singular locus of each restriction of the map. Eleven years later, J.M. Boardman [3] generalized Thom's work to include maps whose singular loci may fail to be manifolds, or whose successive restrictions may fail to be manifolds. In effect, Boardman expanded Thom's work to almost all differential maps on manifolds. The Thom-Boardman classification is realized by an infinite, non-increasing sequence of nonnegative integers referred to as the Thom-Boardman symbol. When the number of nonzero terms is finite, the sequence for the symbol is usually truncated after the last nonzero entry.

Joint work concerning invariants of Gauss maps of theta divisors by M. Adams, C. McCrory, T. Shifrin and R. Varley [1] revealed a fundamental connection between these Gauss maps and secant maps. Continued work by R. Varley (see [5] or Chapter 3 in [7]) indicated a strong potential connection between secant maps and maps defined by the multiplication of two monic single-variable polynomials. The Claim 3.1.2 in [7] is unconditionally true and the transversality hypothesis in Conjecture 3.1.3 in the same paper is also very natural since the geometry of a curve will depend on how "generic" it is embedded. James $\mathrm{M}^{c}$ Kernan [4] proved that for a generic curve of genus $g$ in its canonical embedding, the transversality hypothesis of Janice's conjecture 3.1.3 does hold. Janice and I are still working on a proof of her conjecture 3.1.3. If we succeed, the Thom-Boardman symbol for the secant map of a generic canonically embedded curve and hence the Thom-Boardman symbol for the Gauss map at all smooth points on the theta divisor of a generic Jacobian will be known. So the classification by singularities of these polynomial multiplication maps would result in the classification of the secant maps and hence Gauss maps. However, the

\footnotetext{
*Received July 13, 2011; accepted for publication September 2, 2011.

${ }^{\dagger}$ Department of Mathematics, SUNY Canton, 34 Cornell Drive, Canton, NY 13617, USA (linj@canton.edu).

$\ddagger$ U. S. Department of Defense, 1509 Stevens Creek Drive, North Augusta, SC 29860, USA (janice 1729@yahoo.com).
} 
Thom-Boardman symbol is usually difficult to compute. Even in the case of the polynomial multiplication maps the computation becomes extremely difficult in all but a small number of cases. A conversation with V. Goryunov led R. Varley to conjecture that computing the Thom-Boardman symbol for polynomial multiplication reduces to computing the successive quotients and remainders for the Euclidean algorithm applied to the degrees of the two polynomials.

In her Ph.D. dissertation [7], Janice Wethington revealed many fundamental structures in the Jacobian matrices. She proved Varley's Conjecture in several special cases and obtained upper bounds for the Thom-Boardman symbols. In this paper, we completely prove Varley's Conjecture. Recently, using Theorem 1.2 below, Y. Wang et al. [6] proved that for any non-increasing sequence of nonnegative integers, there is a map-germ with the prescribed sequence as its Thom-Boardman symbol. This result provides a complete list of representatives of map-germs classified by their Thom-Boardman symbols.

For the reader's convenience, let us first recall the definition of Thom-Boardman symbol from [2] and then state Robert Varley's conjecture.

Let $x_{1}, \cdots, x_{m}$ be local coordinates on a differential manifold $M$ of dimension $m$. Denote $\mathcal{A}$ the local ring of germs of differentiable functions at a point $x \in M$. For any ideal $B$ in $\mathcal{A}$, the Jacobian extension, $\Delta_{k} B$, is the ideal spanned by $B$ and all the minors of order $k$ of the Jacobian matrix $\left(\partial \phi_{i} / \partial x_{j}\right)$, denoted $\delta B$, formed from partial derivatives of functions $\phi_{i}$ in $B$. Since the determinant of this matrix is multilinear and since $\left(\partial \phi / \partial x^{\prime}\right)=\partial \phi / \partial x \cdot \partial x / \partial x^{\prime}$, the Jacobian extension is independent of the coordinate system chosen, hence is an invariant of the ideal. We say that $\Delta_{i} B$ is critical if $\Delta_{i} B \neq \mathcal{A}$ but $\Delta_{i-1} B=\mathcal{A}$ (just $\Delta_{1} B \neq \mathcal{A}$ when $i=1$ ). That is, the critical extension of $B$ is $B$ adjoined with the least order minors of the Jacobian matrix of $B$ for which the extension does not coincide with the whole algebra. If every size minor of $\delta B$ is a unit in $\mathcal{A}$, then the map was of full rank at the given point already and the critical extension is the ideal $B$ itself. Note that $B \subseteq \Delta_{i} B$.

Suppose that $N$ is another differential manifold of dimension $n$ and $y_{1}, \cdots, y_{n}$ be local coordinates on it. For a differential map $F: M \rightarrow N, F=\left(f_{1}, f_{2}, \cdots, f_{n}\right)$, we denote $J$ the ideal generated by $f_{1}, \cdots, f_{n}$ in $\mathcal{A}$. Then $\Delta_{k} J$ is spanned by $J$ and all the minors of order $k$ of the Jacobian matrix $\delta J=\left(\partial f_{i} / \partial x_{j}\right)$.

Now we shift the lower indices to upper indices of the critical extensions by the rule $\Delta^{i} J=\Delta_{m-i+1} J$. We repeat the process described above with the resulting ideals until we have a sequence of critical extensions of $J$,

$$
J \subseteq \Delta^{i_{1}} J \subseteq \Delta^{i_{2}} \Delta^{i_{1}} J \subseteq \cdots \subseteq \Delta^{i_{k}} \Delta^{i_{k-1}} \cdots \Delta^{i_{1}} J=\mathbf{m}
$$

where $\mathbf{m}$ is the maximal ideal of $\mathcal{A}$. Then the Thom-Boardman symbol, $T B(J)$, is given by $\left(i_{1}, i_{2}, \cdots, i_{k}\right)$. The purpose of switching the indices is that doing so allows us to express $T B(J)$ as follows:

$$
i_{1}=\operatorname{corank}(J), i_{2}=\operatorname{corank}\left(\Delta^{i_{1}} J\right), \cdots, i_{k}=\operatorname{corank}\left(\Delta^{i_{k-1}} \cdots \Delta^{i_{1}} J\right)
$$

where the rank of ideal is defined to be the maximal number of independent coordinates from the ideal and the corank is the number of variables minus the rank. For concrete examples of computing Thom-Boardman symbols, we refer the interested reader to $[7]$.

Let $M_{n}$ be the set of monic complex polynomials in one variable of degree $n$. $M_{n} \cong \mathbb{C}^{n}$ by the map sending $f(x)=x^{n}+a_{n-1} x^{n-1}+\cdots+a_{0}$ to the $n$-tuple $\left(a_{0}, a_{1}, \cdots, a_{n-1}\right) \in \mathbb{C}^{n}$. 
If we take $f(x)$ of degree $n$ as above and $g(x)=x^{r}+b_{r-1} x^{r-1}+\cdots+b_{0}$ of degree $r$, then the product $h(x)=f(x) g(x)$ is a monic polynomial of the form $h(x)=$ $x^{n+r}+c_{n+r-1} x^{n+r-1}+\cdots+c_{0}$, where the $c_{j}$ 's are polynomials in the coefficients of $f$ and $g$. We can also assume that $n \geq r$. The $c_{j}$ 's are as shown below:

$$
\left\{\begin{array}{l}
c_{n+r-1}=a_{n-1}+b_{r-1} \\
c_{n+r-2}=a_{n-2}+b_{r-2}+a_{n-1} b_{r-1} \\
\text { and } \\
c_{n+r-j}=a_{n-j}+b_{r-j}+\sum_{i+k=n+r-j} a_{i} b_{k}, \text { for } j \leq r \\
c_{n+r-j}=a_{n-j}+\sum_{i+k=n+r-j} a_{i} b_{k}, \text { for } r<j \leq n \\
c_{n+r-j}=\sum_{i+k=n+r-j} a_{i} b_{k}, \text { for } j>n
\end{array} .\right.
$$

This gives us maps

$$
\mu_{n, r}: \mathbb{C}^{n} \times \mathbb{C}^{r} \rightarrow \mathbb{C}^{n+r}
$$

defined by

$$
\left(a_{0}, \cdots, a_{n-1}, b_{0}, \cdots, b_{r-1}\right) \rightarrow\left(c_{n+r-1}, \cdots, c_{0}\right) .
$$

Consider the Euclidean algorithm applied to $n$ and $r$ :

$$
\begin{array}{ll}
n=q_{1} r+r_{1}, & 0<r_{1}<r \\
r=q_{2} r_{1}+r_{2}, & 0<r_{2}<r_{1} \\
\vdots & \\
r_{k-1}=q_{k+1} r_{k}, & 0<r_{k}<r_{k-1}
\end{array}
$$

Let $I(n, r)$ be the tuple given by the Euclidean algorithm on $n$ and $r$ :

$$
I(n, r)=\left(r, \cdots, r, r_{1}, \cdots, r_{1}, \cdots, r_{k}, \cdots, r_{k}\right)
$$

where $r$ is repeated $q_{1}$ times, and $r_{i}$ is repeated $q_{i+1}$ times.

Let $I\left(\mu_{n, r}\right)$ be the ideal in the algebra $\mathcal{A}$ of germs at origin generated by $c_{j}$ 's in the map $\mu_{n, r}: \mathbb{C}^{n} \times \mathbb{C}^{r} \rightarrow \mathbb{C}^{n+r}$. Denote $T B\left(I\left(\mu_{n, r}\right)\right)$ the Thom-Boardman symbol of this ideal, Robert Varley conjectured that

Conjecture 1.1. (Varley's Conjecture) $T B\left(I\left(\mu_{n, r}\right)\right)=I(n, r)$ for any $n \geq r$.

In this paper, we prove Varley's Conjecture, that is, we have

ThEOREM 1.2. $T B\left(I\left(\mu_{n, r}\right)\right)=I(n, r)$ is true for any $n \geq r$.

One of the difficulties in computing Thom-Boardman symbol is that if we simply add all $\left(n+r-i_{j}+1\right)$ minors into the ideal representing the $j$-th critical extension of $I\left(\mu_{n, r}\right)$ the number of generators grows exponentially. In her dissertation, Wethington confirmed Varley's Conjecture for all cases $n+r \leq 10$ by computer. The memory 
demands grew exponentially for those calculations. In this paper, we overcome this difficulty by carefully choosing the generators at each step of the critical extensions. Specifically, we find a group of polynomials such that at each step of the critical extensions we only need to add the same number of polynomials indexed by the corresponding entry in $I(n, r)$. We construct these polynomials explicitly and prove that they have the desired property.

This paper is organized as follows: in section 2 we discuss the first critical extension of $I\left(\mu_{n, r}\right)$ and prove Varley's Conjecture in the special case $n=r$. In section 3 , we first prove some properties of lower Toeplitz matrices and then construct $\left(q_{1} r+r_{1}\right)$ polynomials $\psi_{0}, \cdots, \psi_{q_{1} r-1} ; \psi_{q_{1} r}, \cdots, \psi_{q_{1} r+r_{1}-1}$ explicitly. We show that the $s$-th critical extension of $I\left(\mu_{n, r}\right)$ is exactly obtained from the previous one by adjoining $\psi_{(s-1) r}, \cdots, \psi_{s r-1}$ for $1 \leq s \leq q_{1}$ and the $\left(q_{1}+1\right)$-th critical extension is the $q_{1}$-th one adjoining $\psi_{q_{1} r+1}, \cdots, \psi_{q_{1} r+r_{1}-1}$. Denote $f_{0}(x)=f(x), f_{1}(x)=g(x), r_{-1}=n$ and $r_{0}=r$. Starting from $f_{0}(x)$ and $f_{1}(x)$, we construct a sequence of polynomials $f_{2}(x), \cdots, f_{k+1}(x)$ inductively such that the degree of $f_{i}(x)$ is $r_{i-1}$ and the multiplication of $f_{i}(x)$ and $f_{i+1}(x)$ gives a map $\mu_{r_{i-1}, r_{i}}: \mathbb{C}^{r_{i-1}} \times \mathbb{C}^{r_{i}} \rightarrow \mathbb{C}^{r_{i-1}+r_{i}}$. Following the same idea we can produce $\left(q_{i+1} r_{i}+r_{i+1}\right)$ polynomials with the property that at each of the next $\left(q_{i+1}+1\right)$ steps of the critical extensions of $I\left(\mu_{n, r}\right)$ we only need to add the same number of polynomials indexed by the entries $\left(r_{i}, \cdots, r_{i}, r_{i+1}\right)$ in $I(n, r)$. After adding all such polynomials into $I(n, r)$, we reach the maximal ideal m. Therefore the rest of the entries in $T B\left(I\left(\mu_{n, r}\right)\right)$ are zeros and Varley's Conjecture follows.

Acknowledgment. We appreciate Professor Robert Varley for his detailed explanation about the motivation to compute Thom-Boardman symbols of polynomial multiplication maps. We also thank the referee for a number of helpful comments and suggestions.

2. The first critical extension of $I\left(\mu_{n, r}\right)$. Let $I\left(\mu_{n, r}\right)$ be the ideal generated by $c_{n+r-1}, c_{n+r-2}, \cdots, c_{0}$ defined by the multiplication map $\mu_{n, r}$. There is an interesting fact that becomes obvious when taking the Jacobian $\delta I\left(\mu_{n, r}\right)$. Taking the derivatives of $c_{j}$ 's in descending order from $n+r-1$ to 0 with respect to the $a_{i}$ 's and $b_{i}$ 's in descending order from $n-1$ to 0 and $r-1$ to 0 respectively, we get the following:

$$
\delta I\left(\mu_{n, r}\right)=\left(\begin{array}{cccccccccc}
1 & 0 & 0 & \cdots & \cdots & 0 & 1 & 0 & \cdots & 0 \\
b_{r-1} & 1 & 0 & \cdots & \cdots & 0 & a_{n-1} & 1 & \cdots & 0 \\
b_{r-2} & b_{r-1} & 1 & \cdots & \cdots & \cdots & a_{n-2} & a_{n-1} & \cdots & 0 \\
\vdots & b_{r-2} & b_{r-1} & \cdots & \cdots & \cdots & \vdots & a_{n-2} & \cdots & 1 \\
\vdots & \vdots & b_{r-2} & \cdots & \cdots & 1 & \vdots & \vdots & \cdots & a_{n-1} \\
\vdots & \vdots & \vdots & \cdots & \cdots & b_{r-1} & \vdots & \vdots & \cdots & a_{n-2} \\
b_{0} & \vdots & \vdots & \cdots & \cdots & b_{r-2} & \vdots & \vdots & \cdots & \vdots \\
0 & b_{0} & \vdots & \cdots & \cdots & 0 & \vdots & \vdots & \cdots & \vdots \\
0 & 0 & b_{0} & \cdots & \cdots & \vdots & a_{0} & \vdots & \cdots & \vdots \\
\vdots & \vdots & \vdots & \vdots & \vdots & \vdots & 0 & a_{0} & \cdots & \vdots \\
\vdots & \vdots & \vdots & \vdots & \vdots & \vdots & \vdots & \vdots & \cdots & \vdots \\
0 & 0 & 0 & \cdots & \cdots & b_{0} & 0 & 0 & \cdots & a_{0}
\end{array}\right)
$$


This is the Sylvester matrix for $f$ and $g$. The rank of the Sylvester matrix for two polynomials when evaluated at the origin is the larger of the two degrees and thus the corank is the smaller. This gives the first entry of $T B\left(\mu_{n, r}\right)$ for any $n \geq r$; $i_{1}=\operatorname{corank}\left(\delta I\left(\mu_{n, r}\right)\right)=r$.

Let $d_{n-1}=a_{n-1}-b_{r-1}, d_{n-j}=a_{n-j}-b_{r-j}-\sum_{i+k=j} d_{n-i} b_{r-k}$, for $j \leq r$ and $d_{n-j}=a_{n-j}-\sum_{i+k=j} d_{n-i} b_{r-k}$, for $r<j \leq n$. The following is true.

Proposition 2.1. $\Delta^{r} I\left(\mu_{n, r}\right)=I\left(\mu_{n, r}\right)+\left(d_{0}, \cdots, d_{r-1}\right)$

Proof. By the definition of critical extension, $\Delta^{r} I\left(\mu_{n, r}\right)$ is the sum of $I\left(\mu_{n, r}\right)$ and the ideal spanned by all the $(n+1) \times(n+1)$ minors of $\delta I\left(\mu_{n, r}\right)$. The later one is unchanged under elementary row operations on $\delta I\left(\mu_{n, r}\right)$. We can do row operations on $\delta I\left(\mu_{n, r}\right)$ as follows (in the next section, we will describe these operations in matrix language).

Multiply the first row by $-b_{r-i}$ and add it to the $(i+1)$-th row for $i=1, \cdots, r$. After that, multiply the second row by $-b_{r-i}$ and add it to the $(i+2)$-th row for $i=1, \cdots, r$. Continue this process until all the $b_{0}, \cdots, b_{r-1}$ disappear from the first $n$ columns. After that, multiply the $(n+1)$-th row by $-b_{r-i}$ and add it to the $(n+i+1)$-th row for $i=1, \cdots, r-1$. For each $j=2, \cdots, r-1$, starting from $j=2$, we can multiply the $(n+j)$-th row by $-b_{r-i}$ and add it to the $(n+j+i)$-th row for $i=1, \cdots, r-j$. At the end, we get a matrix with the following form:

$$
\left(\begin{array}{ccccccccc}
1 & 0 & 0 & \cdots & 0 & 1 & 0 & \cdots & 0 \\
0 & 1 & 0 & \ldots & 0 & d_{n-1} & \ddots & \ddots & 0 \\
0 & 0 & 1 & \ldots & \cdots & d_{n-2} & \ddots & \ddots & \vdots \\
\vdots & 0 & \ldots & \ldots & \ldots & \vdots & \ddots & \ddots & 1 \\
\vdots & \vdots & \ldots & \ldots & 1 & \vdots & \ddots & \ddots & \vdots \\
0 & \vdots & \ldots & \ldots & 0 & d_{0} & \ddots & \ddots & d_{r-1} \\
\vdots & \vdots & \vdots & \vdots & \vdots & * & \ddots & \ddots & d_{r-2} \\
\vdots & 0 & \cdots & \ldots & \ldots & * & * & \ddots & \vdots \\
0 & 0 & \cdots & \cdots & 0 & * & * & * & d_{0}
\end{array}\right)
$$

The elements in the position marked with "**" in matrix $(2.2)$ can be generated by $d_{0}, \cdots, d_{r-1}$. Now it is easy to see that the ideal of all the $(n+1) \times(n+1)$ minors of the matrix $(2.2)$ is generated by $d_{0}, \cdots, d_{r-1}$, so is that of $\delta I\left(\mu_{n, r}\right)$. Proposition 2.1 follows.

As an easy consequence of Proposition 2.1, we have the following corollary.

Corollary 2.2. $\operatorname{TB}\left(I\left(\mu_{n, n}\right)\right)=(n)=I(n, n)$ for any positive integer $n$.

Proof. From the discussion on the first Jacobian, we know the first entry in $T B\left(I\left(\mu_{n, n}\right)\right)$ is $n$.

To show that $\operatorname{TB}\left(I\left(\mu_{n, n}\right)\right)=(n)$, we only need to prove that the corank of $\delta \Delta^{n} I\left(\mu_{n, n}\right)$ evaluated at origin is 0 . 
By Proposition $2.1, \Delta^{n} I\left(\mu_{n, n}\right)=I\left(\mu_{n, n}\right)+\left(d_{0}, \cdots, d_{n-1}\right)$, so $\delta \Delta^{n} I\left(\mu_{n, n}\right)$ has the following form when evaluated at origin.

$$
\left(\begin{array}{cc}
I_{n} & I_{n} \\
0 & 0 \\
I_{n} & -I_{n}
\end{array}\right)
$$

whose corank is obviously equal to 0 , hence $T B\left(I\left(\mu_{n, n}\right)\right)=(n)$.

\section{Proof of Theorem 1.2.}

3.1. Toeplitz matrices. Before we give a proof of Theorem 1.2, let us discuss some properties on certain class of matrices called Toeplitz matrices.

A $n \times n$ Toeplitz matrix is a matrix in which each descending diagonal from left to right is constant. The lower shift matrix $L_{n}$ is a $n \times n$ binary matrix with ones only on the subdiagonal and zeros elsewhere. It is obvious that $L_{n}$ is Toeplitz. Moreover, it is nilpotent.

A matrix $V$ is called a lower Toeplitz matrix if $V=v I_{n}+v_{m-1} L_{n}+v_{m-2} L_{n}^{2}+$ $\cdots+v_{0} L_{n}^{m}$ for some $m(m \leq n)$, where $I_{n}$ is the identity matrix and $v, v_{0}, \cdots, v_{m-1}$ are variables or constants.

The following lemma is true.

LEMmA 3.1. Let $V=I_{n}+v_{m-1} L_{n}+v_{m-2} L_{n}^{2}+\cdots+v_{0} L_{n}^{m}$ and $W=I_{n}+$ $w_{l-1} L_{n}+w_{l-2} L_{n}^{2}+\cdots+w_{0} L_{n}^{l}$ be two $n \times n$ lower Toeplitz matrices. Then

1. $V W$ is a lower Toeplitz matrix and $V W=W V$.

2. $V^{-1}$ is a lower Toeplitz matrix and each entry below the diagonal is a polynomial in variables $v_{0}, \cdots, v_{m-1}$.

Proof. It is easy to see that $V W=\left(I_{n}+v_{m-1} L_{n}+v_{m-2} L_{n}^{2}+\cdots+v_{0} L_{n}^{m}\right)\left(I_{n}+\right.$ $\left.w_{l-1} L_{n}+w_{l-2} L_{n}^{2}+\cdots+w_{0} L_{n}^{l}\right)=W V$ and $V W$ is a lower Toeplitz matrix.

Using long division to $\frac{1}{1+v_{m-1} L_{n}+v_{m-2} L_{n}^{2}+\cdots+v_{0} L_{n}^{m}}$ in the formal power series ring $\frac{\mathbb{C}\left[v_{0}, \cdots, v_{m-1}\right]\left[\left[L_{n}\right]\right]}{\left(L_{n}^{n}\right)}$, we immediately have that $V^{-1}$ is a lower Toeplitz matrix and each entry below the diagonal is a polynomial in variables $v_{0}, \cdots, v_{m-1}$.

3.2. Proof of Theorem 1.2. Let $A_{n+r+1}=I_{n+r+1}+a_{n-1} L_{n+r+1}+$ $a_{n-2} L_{n+r+1}^{2}+\cdots+a_{0} L_{n+r+1}^{n}$ and $B_{n+r+1}=I_{n+r+1}+b_{r-1} L_{n+r+1}+b_{r-2} L_{n+r+1}^{2}+$ $\cdots+b_{0} L_{n+r+1}^{r}$, where $a_{i}, b_{j}$ are the coefficients of $f(x)$ and $g(x)$ respectively. The first Jacobian matrix is

$$
\delta I\left(\mu_{n, r}\right)=\left(\left(I_{n+r}, 0\right) B_{n+r+1}\left(\begin{array}{c}
I_{n} \\
0
\end{array}\right),\left(I_{n+r}, 0\right) A_{n+r+1}\left(\begin{array}{c}
I_{r} \\
0
\end{array}\right)\right)
$$

Let $D_{n+r+1}=\left(B_{n+r+1}\right)^{-1} A_{n+r+1}$. It is easy to see that the row operations we did in section 2 on $\delta I\left(\mu_{n, r}\right)$ is exactly multiplying $\delta I\left(\mu_{n, r}\right)$ by $\left(B_{n+r}\right)^{-1}$ on the left, where $B_{n+r}=\left(I_{n+r}, 0\right) B_{n+r+1}\left(\begin{array}{c}I_{n+r} \\ 0\end{array}\right)$. So $D_{n+r+1}=I_{n+r+1}+d_{n-1} L_{n+r+1}+$ $d_{n-2} L_{n+r+1}^{2}+\cdots+d_{0} L_{n+r+1}^{n}+d_{-1} L_{n+r+1}^{n+1}+\cdots+d_{-r} L_{n+r+1}^{n+r}$ for some $d_{-1}, \cdots, d_{-r}$. Comparing the corresponding coefficients $L_{n+r+1}^{n+j}$ for $j=1, \cdots, r$ on both sides of the equation $B_{n+r+1} D_{n+r+1}=A_{n+r+1}$, we have that $d_{-j}+b_{r-1} d_{-j+1}+\cdots+b_{0} d_{r-j}=0$, which imply that $d_{-j}, j=1, \cdots, r$ are generated by $d_{0}, \cdots, d_{r-1}$. This coincides with what we said about the elements in the position marked with "*" in matrix (2.2).

From $A_{n+r+1}=B_{n+r+1} D_{n+r+1}$, it is easy to get the following equations: 


$$
\left\{\begin{array}{l}
a_{n-1}=d_{n-1}+b_{r-1} \\
a_{n-2}=d_{n-2}+b_{r-2}+b_{r-1} d_{n-1} \\
\text { and } \\
a_{n-j}=d_{n-j}+b_{r-j}+\sum_{i+k=j} b_{r-k} d_{n-i}, \text { for } j \leq r \\
a_{n-j}=d_{n-j}+\sum_{i+k=j} b_{r-k} d_{n-i}, \text { for } r<j \leq n
\end{array}\right.
$$

Taking derivatives with respect to $a_{i}$ 's and $b_{i}$ 's in descending order from $n-1$ to 0 and $r-1$ to 0 respectively in the above equations and using the Chain Rule, we have that

$$
\left(\begin{array}{ll}
I_{n}, & 0
\end{array}\right)=\left(\begin{array}{ll}
B, & D
\end{array}\right)\left(\begin{array}{cc}
\left(\frac{\partial d_{i}}{\partial a_{j}}\right) & \left(\begin{array}{c}
\frac{\partial d_{i}}{\partial b_{j}} \\
0
\end{array}\right. \\
I_{r}
\end{array}\right)
$$

where $B=\left(I_{n}, 0\right) B_{n+r+1}\left(\begin{array}{c}I_{n} \\ 0\end{array}\right)$ and $D=\left(I_{n}, 0\right) D_{n+r+1}\left(\begin{array}{c}I_{r} \\ 0\end{array}\right)$. This gives that

$$
\begin{aligned}
B\left(\frac{\partial d_{i}}{\partial a_{j}}\right) & =I_{n} \\
B\left(\frac{\partial d_{i}}{\partial b_{j}}\right)+D & =0 .
\end{aligned}
$$

Let $A=\left(I_{n}, 0\right) A_{n+r+1}\left(\begin{array}{c}I_{r} \\ 0\end{array}\right)$, then $A=\left(I_{n}, 0\right) A_{n+r+1}\left(\begin{array}{c}I_{r} \\ 0\end{array}\right)=$ $\left(I_{n}, 0\right) B_{n+r+1} D_{n+r+1}\left(\begin{array}{c}I_{r} \\ 0\end{array}\right)=(B, 0)\left(\begin{array}{c}D \\ *\end{array}\right)=B D$.

Using Equation (3.3) and $A=B D$, we can prove the following lemma.

LEMMA 3.2. $\left(\frac{\partial^{s} d_{i}}{\partial^{s-1} b_{0} \partial b_{j}}\right)=-s\left(\frac{\partial^{s} d_{i}}{\partial^{s-1} b_{0} \partial a_{j}}\right) D$ for $s=1, \cdots, q_{1}$.

Proof. It is easy to see that $B=\left(I_{n}, 0\right) B_{n+r+1}\left(\begin{array}{c}I_{n} \\ 0\end{array}\right)=I_{n}+b_{r-1} L_{n}+\cdots+b_{0} L_{n}^{r}$ is a lower Toeplitz matrix. Its derivative with respect to $b_{0}$ is again a lower Toeplitz matrix, in fact, $\frac{\partial B}{\partial b_{0}}=L_{n}^{r}$. So $B \frac{\partial B}{\partial b_{0}}=\frac{\partial B}{\partial b_{0}} B$ by Lemma 3.1 or direct verification.

The equation $\frac{\partial B}{\partial b_{0}}=L_{n}^{r}$ implies that any higher derivatives of $B$ with respect to $b_{0}$ is zero. From $B\left(\frac{\partial d_{i}}{\partial a_{j}}\right)=I_{n}$ in Equation (3.3), we have

$$
\left(\frac{\partial d_{i}}{\partial a_{j}}\right)=B^{-1}
$$

Taking derivatives with respect to $b_{0}$ repeatedly on both sides of Equation (3.4) gives

$$
\left(\frac{\partial^{s} d_{i}}{\partial^{s-1} b_{0} \partial a_{j}}\right)=(-1)(-2) \cdots(-s+1) B^{-s}\left(\frac{\partial B}{\partial b_{0}}\right)^{s-1}=(-1)^{s-1}(s-1) ! B^{-s}\left(\frac{\partial B}{\partial b_{0}}\right)^{s-1}
$$

for any positive integer $s$.

From $B\left(\frac{\partial d_{i}}{\partial b_{j}}\right)+D=0$ and $A=B D$, we have

$$
\left(\frac{\partial d_{i}}{\partial b_{j}}\right)=-B^{-1} D=-B^{-2} A
$$


Taking derivatives with respect to $b_{0}$ repeatedly on both sides of Equation (3.6) and using the commutativity $B \frac{\partial B}{\partial b_{0}}=\frac{\partial B}{\partial b_{0}} B$ give that

$$
\left(\frac{\partial^{s} d_{i}}{\partial^{s-1} b_{0} \partial b_{j}}\right)=(-1)(-2) \cdots(-s) B^{-s-1}\left(\frac{\partial B}{\partial b_{0}}\right)^{s-1} A=(-1)^{s} s ! B^{-s}\left(\frac{\partial B}{\partial b_{0}}\right)^{s-1} D \text {. }
$$

Now our lemma follows immediately from Equations (3.5) and (3.7).

We also need the following lemma.

LEMma 3.3. The $n \times r$ matrix $\left(\frac{\partial d_{i}}{\partial b_{j}}\right)$ in Equation (3.3) is the first $r$ columns in a $n \times n$ lower Toeplitz matrix; moreover, the elements $\frac{\partial d_{i}}{\partial b_{j}}$ for $i=0, \cdots, r-1$ and $j=0, \cdots, r-1$ can be generated by $\frac{\partial d_{r-1}}{\partial b_{j}}, j=0, \cdots, r-1$ and $d_{1}, \cdots, d_{r-1}$ if $q_{1} \geq 2$.

Proof. From $B\left(\frac{\partial d_{i}}{\partial b_{j}}\right)+D=0$ in Equation (3.3), we have that $\left(\frac{\partial d_{i}}{\partial b_{j}}\right)=-B^{-1} D$. Let $\widehat{D}=I_{n}+d_{n-1} L_{n}+\cdots+d_{1} L_{n}^{n-1}$, which is a lower Toeplitz matrix. Then $D=$ $\widehat{D}\left(\begin{array}{c}I_{r} \\ 0\end{array}\right)$. By Lemma $3.1,-B^{-1} \widehat{D}$ is a lower Toeplitz matrix. So $\left(\frac{\partial d_{i}}{\partial b_{j}}\right)=-B^{-1} D=$ $-B^{-1} \widehat{D}\left(\begin{array}{c}I_{r} \\ 0\end{array}\right)$ is the first $r$ columns in the $n \times n$ lower Toeplitz matrix $-B^{-1} \widehat{D}$.

Denote $B^{-1} \widehat{D}$ as $I_{n}+t_{n-1} L_{n}+\cdots+t_{1} L_{n}^{n-1}$, we have that

$$
\begin{array}{r}
I_{n}+d_{n-1} L_{n}+\cdots+d_{1} L_{n}^{n-1}=\widehat{D}=B B^{-1} \widehat{D}= \\
\left(I_{n}+b_{r-1} L_{n}+\cdots+b_{0} L_{n}^{r}\right)\left(I_{n}+t_{n-1} L_{n}+\cdots+t_{1} L_{n}^{n-1}\right) .
\end{array}
$$

Comparing the coefficients of $L_{n}^{k}$ for $k=n-r+1, \cdots, n-1$ in Equation (3.8), we have that

$$
d_{i}=t_{i}+b_{r-1} t_{i+1}+\cdots+b_{0} t_{r+i} \text { for } i=1, \cdots, r-1 \text {. }
$$

From Equation (3.9), it is easy to see that $t_{1}, \cdots, t_{r-1}$ are generated by $t_{r}, \cdots, t_{2 r-1}$ and $d_{1}, \cdots, d_{r-1}$. From the equation $\left(\frac{\partial d_{i}}{\partial b_{j}}\right)=-B^{-1} \widehat{D}\left(I_{r}, 0\right)$ we see that $t_{k}=-\frac{\partial d_{i}}{\partial b_{j}}$ for $k=r-j+i$, where $i=0, \cdots, r-1$ and $j=0, \cdots, r-1$. Hence the elements $\frac{\partial d_{i}}{\partial b_{j}}$ for $i=0, \cdots, r-1$ and $j=0, \cdots, r-1$ can be generated by $t_{2 r-1-j}=\frac{\partial d_{r-1}}{\partial b_{j}}, j=0, \cdots, r-1$ and $d_{1}, \cdots, d_{r-1}$. $\mathrm{C}$

As an easy consequence of Lemma 3.2 and Lemma 3.3, we have $q_{1} \geq 2$.

Proposition 3.4. $\Delta^{r}\left(\Delta^{r} I\left(\mu_{n, r}\right)\right)=I\left(\mu_{n, r}\right)+\left(d_{0}, \cdots, d_{r-1}, \frac{\partial d_{r-1}}{\partial b_{r-1}}, \cdots, \frac{\partial d_{r-1}}{\partial b_{0}}\right)$ if

Proof. By Proposition 2.1, we have that $\Delta^{r} I\left(\mu_{n, r}\right)=I\left(\mu_{n, r}\right)+\left(d_{0}, \cdots, d_{r-1}\right)$. To prove this proposition, we only need to show that the corank of $\delta\left(I\left(\mu_{n, r}\right)+\right.$ $\left.\left(d_{0}, \cdots, d_{r-1}\right)\right)$ evaluated at origin is $r$ and

$$
\Delta^{r}\left(I\left(\mu_{n, r}\right)+\left(d_{0}, \cdots, d_{r-1}\right)\right)=I\left(\mu_{n, r}\right)+\left(d_{0}, \cdots, d_{r-1}, \frac{\partial d_{r-1}}{\partial b_{r-1}}, \cdots, \frac{\partial d_{r-1}}{\partial b_{0}}\right)
$$




$$
\begin{aligned}
& \text { Because } \delta\left(I\left(\mu_{n, r}\right)+\left(d_{0}, \cdots, d_{r-1}\right)\right)=\left(\begin{array}{c}
\delta\left(I\left(\mu_{n, r}\right)\right) \\
\left(\frac{\partial d_{i}}{\partial a_{j}}\right),\left(\frac{\partial d_{i}}{\partial b_{j}}\right)
\end{array}\right)=
\end{aligned}
$$

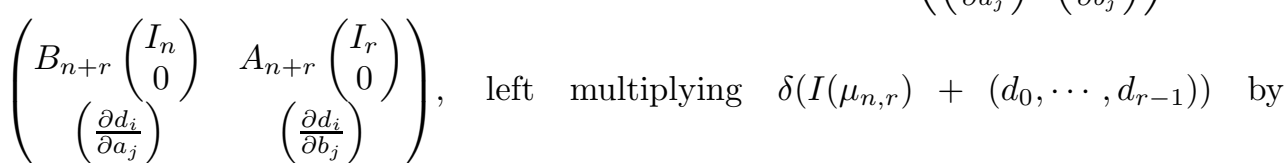

$$
\begin{aligned}
& \left(\begin{array}{ccc}
I_{n} & 0 & 0 \\
0 & I_{r} & 0 \\
-\left(\frac{\partial d_{i}}{\partial a_{j}}\right) & 0 & I_{r}
\end{array}\right)\left(\begin{array}{cc}
\left(B_{n+r}\right)^{-1} & 0 \\
0 & I_{r}
\end{array}\right) \text { gives } \\
& \left(\begin{array}{cc}
I_{n} & D \\
0 & * \\
0 & -\left(\frac{\partial d_{i}}{\partial a_{j}}\right) D+\left(\frac{\partial d_{i}}{\partial b_{j}}\right)
\end{array}\right)
\end{aligned}
$$

where elements in the position marked by "**" can be generated by $d_{0}, \cdots, d_{r-1}$.

By Lemma 3.2 (the case $s=1$ ), we can rewrite the above matrix as

$$
\left(\begin{array}{cc}
I_{n} & D \\
0 & * \\
0 & 2\left(\frac{\partial d_{i}}{\partial b_{j}}\right)
\end{array}\right)
$$

The $*$ part is given by $\left(0, I_{r}\right)\left(B_{n+r}\right)^{-1} A_{n+r}\left(\begin{array}{c}I_{r} \\ 0\end{array}\right)$, which evaluated at origin is $\left(0, I_{r}\right)\left(\begin{array}{c}I_{r} \\ 0\end{array}\right)=0$ because $q_{1} \geq 2$. The same argument gives that $\left(\frac{\partial d_{i}}{\partial b_{j}}\right)=$ $-\left(0, I_{r}\right) B^{-1} \widehat{D}\left(\begin{array}{c}I_{r} \\ 0\end{array}\right)$ evaluated at origin is also equal to $\left(0, I_{r}\right)\left(\begin{array}{c}I_{r} \\ 0\end{array}\right)=0$. Therefor the corank of $\delta\left(I\left(\mu_{n, r}\right)+\left(d_{0}, \cdots, d_{r-1}\right)\right)$ evaluated at origin is $r$.

By Lemma 3.3, any element in the $r \times r$ matrix $\left(\frac{\partial d_{i}}{\partial b_{j}}\right)$ can be generated by $\frac{\partial d_{r-1}}{\partial b_{j}}, j=0, \cdots, r-1$ and $d_{1}, \cdots, d_{r-1}$. So any $(n+1) \times(n+1)$ minor of the matrix in $(3.12)$ can be generated by $\frac{\partial d_{r-1}}{\partial b_{j}}, j=0, \cdots, r-1$ and $d_{0}, \cdots, d_{r-1}$ because it must have at least one row whose elements are from $*$ or $2\left(\frac{\partial d_{i}}{\partial b_{j}}\right)$. This implies that

$$
\Delta^{r}\left(I\left(\mu_{n, r}\right)+\left(d_{0}, \cdots, d_{r-1}\right)\right) \subseteq I\left(\mu_{n, r}\right)+\left(d_{0}, \cdots, d_{r-1}, \frac{\partial d_{r-1}}{\partial b_{r-1}}, \cdots, \frac{\partial d_{r-1}}{\partial b_{0}}\right)
$$

For each $j=0, \cdots, r-1$, the $(n+1) \times(n+1)$ minor $\left(\begin{array}{cc}I_{n} & \# \\ 0 & 2 \frac{\partial d_{r-1}}{\partial b_{j}}\end{array}\right)$ has determinant $2 \frac{\partial d_{r-1}}{\partial b_{j}}$, so the $\subseteq$ in Equation (3.13) is actually an equality. This proves Proposition 3.4. $\textrm{ }$

Let $\psi_{i}=d_{i}$ for $i=0, \cdots, r-1$ and $\psi_{s r+i}=\frac{\partial^{s} d_{r-1}}{\partial^{s-1} b_{0} \partial b_{r-1-i}}$ for $i=0, \cdots, r-1$ and $s=1, \cdots, q_{1}-1$. We have the following lemma.

LEMMA 3.5. $\left(\frac{\partial \psi_{s r+i}}{\partial b_{j}}\right)=-(s+1)\left(\frac{\partial \psi_{s r+i}}{\partial a_{j}}\right) D$ for $s=0, \cdots, q_{1}-1$. 
Proof. Equation (3.3) implies that $\left(\frac{\partial d_{i}}{\partial a_{j}}\right)=B^{-1}$ and $\left(\frac{\partial d_{i}}{\partial b_{j}}\right)=-B^{-1} D$. So $\left(\frac{\partial d_{i}}{\partial b_{j}}\right)=-\left(\frac{\partial d_{i}}{\partial a_{j}}\right) D$. Lemma 3.5 is true in the case $s=0$.

For $s \geq 1$, by Lemma 3.3 we have that $\frac{\partial d_{r-1}}{\partial b_{r-1-i}}=\frac{\partial d_{i}}{\partial b_{0}}$, so $\psi_{s r+i}=\frac{\partial^{s} d_{r-1}}{\partial^{s-1} b_{0} \partial b_{r-1-i}}=$ $\frac{\partial^{s-1}}{\partial^{s-1} b_{0}}\left(\frac{\partial d_{r-1}}{\partial b_{r-1-i}}\right)=\frac{\partial^{s-1}}{\partial^{s-1} b_{0}}\left(\frac{\partial d_{i}}{\partial b_{0}}\right)=\frac{\partial^{s} d_{i}}{\partial^{s} b_{0}}$.

By Lemma 3.2 we have that $\left(\frac{\partial \psi_{s r+i}}{\partial b_{j}}\right)=\left(\frac{\partial}{\partial b_{j}} \frac{\partial^{s} d_{i}}{\partial^{s} b_{0}}\right)=\frac{\partial}{\partial b_{0}}\left(\frac{\partial^{s} d_{i}}{\partial^{s-1} b_{0} \partial b_{j}}\right)=$ $\frac{\partial}{\partial b_{0}}\left(-s \frac{\partial^{s} d_{i}}{\partial^{s-1} b_{0} \partial a_{j}} D\right)=-s\left(\frac{\partial}{\partial a_{j}} \frac{\partial^{s} d_{i}}{\partial^{s} b_{0}}\right) D-s\left(\frac{\partial^{s} d_{i}}{\partial^{s-1} b_{0} \partial a_{j}}\right) \frac{\partial D}{\partial b_{0}}$. Our lemma follows if we can show that $-s\left(\frac{\partial^{s} d_{i}}{\partial^{s-1} b_{0} \partial a_{j}}\right) \frac{\partial D}{\partial b_{0}}=-\left(\frac{\partial}{\partial a_{j}} \frac{\partial^{s} d_{i}}{\partial^{s} b_{0}}\right) D$. This can be done as follows.

From the equation $A=B D$, we have that $\frac{\partial B}{\partial b_{0}} D+B \frac{\partial D}{\partial b_{0}}=0$. So $\frac{\partial D}{\partial b_{0}}=-B^{-1} \frac{\partial B}{\partial b_{0}} D$. Applying Equation (3.5) to both indices $s$ and $s+1$, we have that $-s\left(\frac{\partial^{s} d_{i}}{\partial^{s-1} b_{0} \partial a_{j}}\right) \frac{\partial D}{\partial b_{0}}=$ $s\left(\frac{\partial^{s} d_{i}}{\partial^{s-1} b_{0} \partial a_{j}}\right) B^{-1} \frac{\partial B}{\partial b_{0}} D=(-1)^{s-1} s ! B^{-(s+1)}\left(\frac{\partial B}{\partial b_{0}}\right)^{s} D=-\left(\frac{\partial}{\partial a_{j}} \frac{\partial^{s} d_{i}}{\partial^{s} b_{0}}\right) D$. This completes the proof of Lemma 3.5 .

TheOREM 3.6. $\overbrace{\Delta^{r} \cdots \Delta^{r}}^{s} I\left(\mu_{n, r}\right)=I\left(\mu_{n, r}\right)+\left(\psi_{0}, \cdots, \psi_{s r-1}\right)$ for $s=1, \cdots, q_{1}$.

Proof. The case $s=1$ has been proved in Proposition 2.1. If $q_{1}=1$, we are done. So we may assume that $q_{1} \geq 2$.

Suppose that Theorem 3.6 is true for $s=1, \cdots, p$. By Proposition 2.1 and Proposition 3.4, we may assume $p \geq 2$. If $p=q_{1}$, we are done. Otherwise we may assume that $p \leq q_{1}-1$.

By the inductive assumption, we have that

$$
\overbrace{\Delta^{r} \cdots \Delta^{r}}^{p} I\left(\mu_{n, r}\right)=I\left(\mu_{n, r}\right)+\left(\psi_{0}, \cdots, \psi_{p r-1}\right) .
$$

We need to prove that the corank of $\delta\left(I\left(\mu_{n, r}\right)+\left(\psi_{0}, \cdots, \psi_{p r-1}\right)\right)$ evaluated at origin is $r$ and

$$
\Delta^{r}(\overbrace{\Delta^{r} \cdots \Delta^{r}}^{p} I\left(\mu_{n, r}\right))=\Delta^{r}\left(I\left(\mu_{n, r}\right)+\left(\psi_{0}, \cdots, \psi_{p r-1}\right)\right)=I\left(\mu_{n, r}\right)+\left(\psi_{0}, \cdots, \psi_{(p+1) r-1}\right) .
$$

It is easy to see that $\delta\left(I\left(\mu_{n, r}\right)+\left(\psi_{0}, \cdots, \psi_{p r-1}\right)\right)=\left(\begin{array}{c}\delta\left(I\left(\mu_{n, r}\right)\right) \\ \left(\frac{\partial \psi_{s r+i}}{\partial a_{j}}, \frac{\partial \psi_{s r+i}}{\partial b_{j}}\right)\end{array}\right)$, where $s$ varies from 0 to $p-1$ and $i$ from 0 to $r-1$ respectively. Left multiplying $\delta\left(I\left(\mu_{n, r}\right)+\right.$ $\left.\left(\psi_{0}, \cdots, \psi_{p r-1}\right)\right)$ by $\left(\begin{array}{cccc}\left(\begin{array}{cc}I_{n} & 0 \\ 0 & I_{r}\end{array}\right) & 0 & \cdots & 0 \\ -\left(\frac{\partial \psi_{i}}{\partial a_{j}}\right) & I_{r} & \cdots & 0 \\ \vdots & \vdots & \ddots & \vdots \\ -\frac{\partial \psi_{(p-1) r+i}}{\partial a_{j}} & 0 & \cdots & I_{r}\end{array}\right)\left(\begin{array}{cccc}\left(B_{n+r}\right)^{-1} & 0 & \cdots & 0 \\ 0 & I_{r} & \cdots & \vdots \\ \vdots & \ddots & \ddots & 0 \\ 0 & \cdots & 0 & I_{r}\end{array}\right)$, we get the following matrix

$$
\left(\begin{array}{cc}
I_{n} & D \\
0 & * \\
0 & -\left(\frac{\partial \psi_{s r+i}}{\partial a_{j}}\right) \\
& D+\left(\frac{\partial \psi_{s r+i}}{\partial b_{j}}\right)
\end{array}\right)
$$


where elements in the position marked by "**" can be generated by $d_{0}, \cdots, d_{r-1}$ and $s$ varies from 0 to $p-1$.

By Lemma 3.5 and induction assumption, it is equal to

$$
\left(\begin{array}{cc}
I_{n} & D \\
0 & * \\
0 & \left(\frac{1}{p}+1\right)\left(\frac{\partial \psi_{(p-1) r+i}}{\partial b_{j}}\right)
\end{array}\right)
$$

where elements in the position marked by "**" can be generated by $\psi_{0}, \cdots, \psi_{p r-1}$.

By induction assumption, the corank of $\left(\begin{array}{cc}I_{n} & D \\ 0 & *\end{array}\right)$ evaluated at origin is $r$. To show that the corank of $\delta\left(I\left(\mu_{n, r}\right)+\left(\psi_{0}, \cdots, \psi_{p r-1}\right)\right)$ evaluated at origin is $r$, it is sufficient to prove $\left(\frac{\partial \psi_{(p-1) r+i}}{\partial b_{j}}\right)$ is zero when evaluated at origin.

The matrix $\left(\frac{\partial \psi_{(p-1) r+i}}{\partial b_{j}}\right)=\left(\frac{\partial}{\partial b_{j}}\left(\frac{\partial^{p-1} d_{r-1}}{\partial{ }^{p-2} b_{0} \partial b_{r-1-i}}\right)\right)=\left(\frac{\partial}{\partial b_{j}}\left(\frac{\partial^{p-2}}{\partial p-2 b_{0}} \frac{\partial d_{r-1}}{\partial b_{r-1-i}}\right)\right)=$ $\left(\frac{\partial}{\partial b_{j}}\left(\frac{\partial^{p-2}}{\partial^{p-2} b_{0}} \frac{\partial d_{i}}{\partial b_{0}}\right)\right)=\left(\frac{\partial^{p-1}}{\partial^{p-1} b_{0}}\left(\frac{\partial d_{i}}{\partial b_{j}}\right)\right)$. By Equation (3.6), we have that $\left(\frac{\partial^{p-1}}{\partial^{p-1} b_{0}}\left(\frac{\partial d_{i}}{\partial b_{j}}\right)\right)=-\left(0, I_{r}\right)\left(\frac{\partial^{p-1}}{\partial^{p-1} b_{0}}\left(B^{-2}\right)\right) A=(-1)^{p} p !\left(0, I_{r}\right) B^{-p-1}\left(\frac{\partial B}{\partial b_{0}}\right)^{p-1} A=$ $(-1)^{p} p !\left(0, I_{r}\right) B^{-p-1} L_{n}^{(p-1) r} A$, which evaluated at origin is zero because $B^{-p-1} L_{n}^{(p-1) r} A$ evaluated at origin has the form $\left(\begin{array}{c}0 \\ I_{r} \\ 0\end{array}\right)$ and the bottom 0 consists of $\left(q_{1}-p\right) r \geq r$ rows.

By the definition $\frac{\partial \psi_{(p-1) r+i}}{\partial b_{j}}=\frac{\partial}{\partial b_{j}}\left(\frac{\partial^{p-1} d_{r-1}}{\partial^{p-2} b_{0} \partial b_{r-1-i}}\right)=\frac{\partial}{\partial b_{j}}\left(\frac{\partial^{p-2}}{\partial^{p-2} b_{0}} \frac{\partial d_{r-1}}{\partial b_{r-1-i}}\right)=$ $\frac{\partial}{\partial b_{j}}\left(\frac{\partial^{p-2}}{\partial^{p-2} b_{0}} \frac{\partial d_{i}}{\partial b_{0}}\right)=\frac{\partial}{\partial b_{0}} \frac{\partial^{p-2}}{\partial^{p-2} b_{0}}\left(\frac{\partial d_{i}}{\partial b_{j}}\right)=\frac{\partial}{\partial b_{0}} \frac{\partial \psi_{(p-2) r+i}}{\partial b_{j}}$. By induction assumption $\frac{\partial \psi_{(p-2) r+i}}{\partial b_{j}}$ can be generated by $\psi_{0}, \cdots, \psi_{(p-1) r+r-1}$ and $\frac{\partial \psi_{(p-1) r+i}}{\partial b_{0}}$ for $i=0, \cdots, r-1$. By the definition $\frac{\partial \psi_{(p-1) r+i}}{\partial b_{0}}=\frac{\partial}{\partial b_{0}}\left(\frac{\partial^{p-1} d_{r-1}}{\partial^{p-2} b_{0} \partial b_{r-1-i}}\right)=\frac{\partial^{p} d_{r-1}}{\partial^{p-1} b_{0} \partial b_{r-1-i}}=\psi_{p r+i}$. Therefore any $(n+1) \times(n+1)$ minor of matrix (3.16) and hence $\delta\left(I\left(\mu_{n, r}\right)+\left(\psi_{0}, \cdots, \psi_{p r-1}\right)\right)$ can be generated by $\left(\psi_{0}, \cdots, \psi_{(p+1) r-1}\right)$, this implies that $\Delta^{r}\left(I\left(\mu_{n, r}\right)+\left(\psi_{0}, \cdots, \psi_{p r-1}\right)\right) \subseteq$ $I\left(\mu_{n, r}\right)+\left(\psi_{0}, \cdots, \psi_{(p+1) r-1}\right)$. Actually the inequality is an equality because each $\psi_{p r+i}$ is only different from a $(n+1) \times(n+1)$ minor of $\delta\left(I\left(\mu_{n, r}\right)+\left(\psi_{0}, \cdots, \psi_{p r-1}\right)\right)$ by a nonzero constant. Theorem 3.6 follows.

As an easy corollary of Theorem 3.6 and its proof, we have

Corollary 3.7. The first $q_{1}$ entries in $T B\left(I\left(\mu_{n, r}\right)\right)$ are $(r, \cdots, r)$.

Our next goal is to prove that

Proposition 3.8. The $\left(q_{1}+1\right)$-th entry in TB $\left(I\left(\mu_{n, r}\right)\right)$ is $r_{1}$.

Proof. By Theorem 3.6, it is sufficient to prove that the rank of $\delta\left(I\left(\mu_{n, r}\right)+\right.$ $\left.\left(\psi_{0}, \cdots, \psi_{q_{1} r-1}\right)\right)$ evaluated at origin is $n+r-r_{1}$. By the proof of Theorem 3.6, we only need to prove that the rank of $\left(\frac{\partial \psi_{\left(q_{1}-1\right) r+i}}{\partial b_{j}}\right)$ evaluated at origin is $r-r_{1}$, where $i=0, \cdots, r-1$ and $j=0, \cdots, r-1$.

When $q_{1}=1$, we have that $\left(\frac{\partial \psi_{\left(q_{1}-1\right) r+i}}{\partial b_{j}}\right)=\left(\frac{\partial d_{i}}{\partial b_{j}}\right)$. By Equation (3.6) and that $B^{-1}$ is equal to $I_{n}$ when evaluated at origin, we have that $\left(\frac{\partial d_{i}}{\partial b_{j}}\right)$ evaluated at origin 
has the same rank as that of $-\left(\begin{array}{ll}0 & I_{r}\end{array}\right)\left(\begin{array}{c}I_{r} \\ 0\end{array}\right)$, the latter one has rank $r-r_{1}$ because $n=r_{1}+r$ and $\left(\begin{array}{ll}0 & I_{r}\end{array}\right)\left(\begin{array}{c}I_{r} \\ 0\end{array}\right)$ represents the first $r$ columns in the $r \times n$ matrix $\left(\begin{array}{ll}0 & I_{r}\end{array}\right)$.

When $q_{1}>1$, we have that $\left(\frac{\partial \psi_{\left(q_{1}-1\right) r+i}}{\partial b_{j}}\right)=\left(\frac{\partial}{\partial b_{j}} \frac{\partial^{q_{1}-1} d_{r-1}}{\partial q_{1}-2 b_{0} \partial b_{r-1-i}}\right)=$ $\left(\frac{\partial}{\partial b_{j}} \frac{\partial^{q_{1}-2}}{\partial^{q_{1}-2} b_{0}} \frac{\partial d_{r-1}}{\partial b_{r-1-i}}\right)=\left(\frac{\partial}{\partial b_{j}} \frac{\partial^{q_{1}-2}}{\partial^{q_{1}-2} b_{0}} \frac{\partial d_{i}}{\partial b_{0}}\right)=\left(\frac{\partial^{q_{1}} d_{i}}{\partial^{q_{1}-1} b_{0} \partial b_{j}}\right)$. By Equation (3.7), we have that $\left(\frac{\partial^{q_{1}} d_{i}}{\partial^{q_{1}-1} b_{0} \partial b_{j}}\right)=\left(\begin{array}{ll}0 & I_{r}\end{array}\right)(-1)^{q_{1}} q_{1} ! B^{-q_{1}}\left(\frac{\partial B}{\partial b_{0}}\right)^{q_{1}-1} D$, which evaluated at origin has the same rank as $\left(\begin{array}{lll}0 & 0 & I_{r}\end{array}\right) L_{n}^{\left(q_{1}-1\right) r}\left(\begin{array}{c}I_{r} \\ 0 \\ 0\end{array}\right)$, where the first zero in the $r \times n$ matrix $\left(\begin{array}{lll}0 & 0 & I_{r}\end{array}\right)$ represents the first $\left(q_{1}-1\right) r$ columns and the second zero represents the next $r_{1}$ columns. Because $L_{n}^{\left(q_{1}-1\right) r}=\left(\begin{array}{ccc}0 & 0 & 0 \\ I_{r_{1}} & 0 & 0 \\ 0 & I_{r} & 0\end{array}\right)$, so $\left(\begin{array}{lll}0 & 0 & I_{r}\end{array}\right) L_{n}^{\left(q_{1}-1\right) r}=\left(\begin{array}{lll}0 & I_{r} & 0\end{array}\right)$, where the first zero in $\left(\begin{array}{lll}0 & I_{r} & 0\end{array}\right)$ occupies the first $r_{1}$ columns and the second one occupies the last $\left(q_{1}-1\right) r$ columns. It is easy to see that $\left(\begin{array}{lll}0 & 0 & I_{r}\end{array}\right) L_{n}^{\left(q_{1}-1\right) r}\left(\begin{array}{c}I_{r} \\ 0 \\ 0\end{array}\right)=\left(\begin{array}{lll}0 & I_{r} & 0\end{array}\right)\left(\begin{array}{c}I_{r} \\ 0 \\ 0\end{array}\right)$ has rank $r-r_{1}$.

In order to obtain the $\left(q_{1}+1\right)$-th critical extension $\Delta^{r_{1}}(\overbrace{\Delta^{r} \cdots \Delta^{r}}^{q_{1}} I\left(\mu_{n, r}\right))$, we need a key lemma.

Denote $B^{-q_{1}} \widehat{D}=I_{n}+\alpha_{n-1} L_{n}+\cdots+\alpha_{1} L_{n}^{n-1}$. We have that

$\left(\begin{array}{ll}0 & I_{r}\end{array}\right) L_{n}^{\left(q_{1}-1\right) r} B^{-q_{1}} \widehat{D}\left(\begin{array}{c}I_{r} \\ 0\end{array}\right)=\left(\begin{array}{cccccc}\alpha_{q_{1} r} & \cdots & \alpha_{n-1} & 1 & \cdots & 0 \\ \vdots & \cdots & \cdots & \alpha_{n-1} & \ddots & \vdots \\ \vdots & \cdots & \cdots & \vdots & \cdots & 1 \\ \vdots & \vdots & \vdots & & & \\ \alpha_{\left(q_{1}-1\right) r+1} & \cdots & \alpha_{n-r} & \alpha_{n-r+1} & \cdots & \alpha_{q_{1} r}\end{array}\right)$

It is easy to see that

$\left(\begin{array}{ll}0 & I_{r}\end{array}\right) L_{n}^{\left(q_{1}-1\right) r} B^{-q_{1}} \widehat{D}\left(\begin{array}{c}I_{r} \\ 0\end{array}\right)\left(\begin{array}{c}0 \\ I_{r-r_{1}}\end{array}\right) \quad=\quad\left(\begin{array}{llll}I_{r} & + & \alpha_{n-1} L_{r} & +\end{array}\right]+$ $\left.\alpha_{n-r+1} L_{r}^{r-1}\right)\left(\begin{array}{c}I_{r-r_{1}} \\ 0\end{array}\right)$

The matrix $\left(I_{r}+\alpha_{n-1} L_{r}+\cdots+\alpha_{n-r+1} L_{r}^{r-1}\right)^{-1}\left(0, I_{r}\right) L_{n}^{\left(q_{1}-1\right) r} B^{-q_{1}} \widehat{D}\left(\begin{array}{c}I_{r} \\ 0\end{array}\right)$ has the form

$$
\left(\begin{array}{cc}
* & I_{r-r_{1}} \\
K & 0
\end{array}\right)
$$

where $K=\left(0, I_{r_{1}}\right)\left(I_{r}+\alpha_{n-1} L_{r}+\cdots+\alpha_{n-r+1} L_{r}^{r-1}\right)^{-1}\left(0, I_{r}\right) L_{n}^{\left(q_{1}-1\right) r} B^{-q_{1}} \widehat{D}\left(\begin{array}{c}I_{r} \\ 0\end{array}\right)\left(\begin{array}{c}I_{r_{1}} \\ 0\end{array}\right)$ is a $r_{1} \times r_{1}$ matrix.

We have the following lemma.

Lemma 3.9. The elements in the first row of $K$ and $\psi_{0}, \cdots, \psi_{q_{1} r-1}$ generate all elements in $K$. 
Proof. Denote $\Phi=I_{r}+\alpha_{n-1} L_{r}+\cdots+\alpha_{n-r+1} L_{r}^{r-1}$ and $\left(I_{n}+\alpha_{n-1} L_{n}+\cdots+\right.$ $\left.\alpha_{1} L_{n}^{n-1}\right)^{-1}=I_{n}+\beta_{n-1} L_{n}+\cdots+\beta_{1} L_{n}^{n-1}$. Using the partition $\left(r_{1}, r,\left(q_{1}-1\right) r\right)$ of $n$, we can split $I_{n}+\beta_{n-1} L_{n}+\cdots+\beta_{1} L_{n}^{n-1}$ and $I_{n}+\alpha_{n-1} L_{n}+\cdots+\alpha_{1} L_{n}^{n-1}$ into $3 \times 3$ block matrices. Comparing the $(2,1)$ block in $\left(I_{n}+\beta_{n-1} L_{n}+\cdots+\beta_{1} L_{n}^{n-1}\right)\left(I_{n}+\right.$ $\left.\alpha_{n-1} L_{n}+\cdots+\alpha_{1} L_{n}^{n-1}\right)=I_{n}$ we have that

$$
\left(\begin{array}{ccc}
\beta_{q_{1} r} & \cdots & \beta_{n-1} \\
\vdots & \vdots & \vdots \\
\beta_{\left(q_{1}-1\right) r+1} & \cdots & \beta_{n-r}
\end{array}\right)\left(\begin{array}{ccc}
1 & \cdots & 0 \\
\vdots & \ddots & \vdots \\
\alpha_{n-r_{1}+1} & \cdots & 1
\end{array}\right)+\Phi^{-1}\left(\begin{array}{ccc}
\alpha_{q_{1} r} & \cdots & \alpha_{n-1} \\
\vdots & \vdots & \vdots \\
\alpha_{\left(q_{1}-1\right) r+1} & \cdots & \alpha_{n-r}
\end{array}\right)=0 .
$$

So

$$
\begin{gathered}
K=\left(0, I_{r_{1}}\right) \Phi^{-1}\left(\begin{array}{ccc}
\alpha_{q_{1} r} & \cdots & \alpha_{n-1} \\
\vdots & \vdots & \vdots \\
\alpha_{\left(q_{1}-1\right) r+1} & \cdots & \alpha_{n-r}
\end{array}\right)=-\left(0, I_{r_{1}}\right)\left(\begin{array}{ccc}
\beta_{q_{1} r} & \cdots & \beta_{n-1} \\
\vdots & \vdots & \vdots \\
\beta_{\left(q_{1}-1\right) r+1} & \cdots & \beta_{n-r}
\end{array}\right) \\
\left(\begin{array}{ccc}
1 & \cdots & 0 \\
\vdots & \ddots & \vdots \\
\alpha_{n-r_{1}+1} & \cdots & 1
\end{array}\right)=-\left(\begin{array}{ccc}
\beta_{\left(q_{1}-1\right) r+r_{1}} & \cdots & \beta_{\left(q_{1}-1\right) r+2 r_{1}-1} \\
\vdots & \vdots & \vdots \\
\beta_{\left(q_{1}-1\right) r+1} & \cdots & \beta_{\left(q_{1}-1\right) r+r_{1}}
\end{array}\right)\left(\begin{array}{ccc}
1 & & \\
\vdots & \ddots & \vdots \\
\alpha_{n-r_{1}+1} & \cdots & 1
\end{array}\right)
\end{gathered}
$$

Because each row in $K$ can be generated by the corresponding row $\operatorname{in}\left(\begin{array}{ccc}\beta_{\left(q_{1}-1\right) r+r_{1}} & \cdots & \beta_{\left(q_{1}-1\right) r+2 r_{1}-1} \\ \vdots & \vdots & \vdots \\ \beta_{\left(q_{1}-1\right) r+1} & \cdots & \beta_{\left(q_{1}-1\right) r+r_{1}}\end{array}\right)$ and vice versa, to prove Lemma 3.9 , it is sufficient to show that $\beta_{\left(q_{1}-1\right) r+r_{1}}, \cdots, \beta_{\left(q_{1}-1\right) r+2 r_{1}-1}$ and $\psi_{0}, \cdots, \psi_{q_{1} r-1}$ generate all $\beta_{\left(q_{1}-1\right) r+i}$ for $1 \leq i \leq r_{1}-1$.

Denote $B^{-\left(q_{1}-1\right)} \widehat{D}=B^{-q_{1}} \widehat{A}$ as $I_{n}+\gamma_{n-1} L_{n}+\cdots+\gamma_{1} L_{n}^{n-1}$, where $\widehat{A}=B \widehat{D}$. From Equation (3.7), we have that $\gamma_{\left(q_{1}-1\right) r+i} \propto \frac{\partial^{q_{1}-1} d_{r-1}}{\partial^{q_{1}-2} b_{0} \partial b_{r-1-i}}=\psi_{\left(q_{1}-1\right) r+i}$ for $i=$ $0, \cdots, r-1$.

From the equation $\left(I_{n}+\alpha_{n-1} L_{n}+\cdots+\alpha_{1} L_{n}^{n-1}\right)^{-1}=B^{q_{1}} \widehat{D}^{-1}$, we have that $B^{-\left(q_{1}-1\right)} \widehat{D}\left(I_{n}+\alpha_{n-1} L_{n}+\cdots+\alpha_{1} L_{n}^{n-1}\right)^{-1}=B$, that is,

$$
\left(I_{n}+\gamma_{n-1} L_{n}+\cdots+\gamma_{1} L_{n}^{n-1}\right)\left(I_{n}+\beta_{n-1} L_{n}+\cdots+\beta_{1} L_{n}^{n-1}\right)=I_{n}+b_{r-1} L_{n}+\cdots+b_{0} L_{n}^{r}
$$

Comparing the coefficients of $L_{n}^{k}$ for $k=r+1, \cdots, r+r_{1}-1$ in both sides of Equation (3.17), we have that

$$
\begin{aligned}
& \beta_{\left(q_{1}-1\right) r+i}+\beta_{\left(q_{1}-1\right) r+i+1} \gamma_{n-1}+\cdots+\beta_{n-1} \gamma_{\left(q_{1}-1\right) r+i+1}+\gamma_{\left(q_{1}-1\right) r+i}=0 \\
& \text { for } i=r_{1}-1, \cdots, 1 .
\end{aligned}
$$

For each term $\beta_{k} \gamma_{l}$ in the equation $\beta_{\left(q_{1}-1\right) r+r_{1}-1}+\beta_{\left(q_{1}-1\right) r+r_{1}} \gamma_{n-1}+\cdots+$ $\beta_{n-1} \gamma_{\left(q_{1}-1\right) r+r_{1}}+\gamma_{\left(q_{1}-1\right) r+r_{1}-1}=0$, we have either $\left(q_{1}-1\right) r+r_{1} \leq k \leq$ $\left(q_{1}-1\right) r+2 r_{1}-1$ or $\left(q_{1}-1\right) r+r_{1} \leq l=n+\left(q_{1}-1\right) r+r_{1}-1-k \leq$ $n+\left(q_{1}-1\right) r+r_{1}-1-\left(q_{1}-1\right) r-2 r_{1}=q_{1} r-1=\left(q_{1}-1\right) r+r-1$. So $\beta_{\left(q_{1}-1\right) r+r_{1}-1}$ can be generated by $\beta_{\left(q_{1}-1\right) r+r_{1}}, \cdots, \beta_{\left(q_{1}-1\right) r+2 r_{1}-1}$ and $\gamma_{\left(q_{1}-1\right) r+r_{1}}=$ $\psi_{\left(q_{1}-1\right) r+r_{1}}, \cdots, \gamma_{\left(q_{1}-1\right) r+r-1}=\psi_{\left(q_{1}-1\right) r+r-1}$. Using Equation (3.18) and induction on $i$ in descend order, we can prove that $\beta_{\left(q_{1}-1\right) r+r_{1}}, \cdots, \beta_{\left(q_{1}-1\right) r+2 r_{1}-1}$ and 
$\gamma_{\left(q_{1}-1\right) r+1}=\psi_{\left(q_{1}-1\right) r+1}, \cdots, \gamma_{\left(q_{1}-1\right) r+r-1}=\psi_{\left(q_{1}-1\right) r+r-1}$ generate all $\beta_{\left(q_{1}-1\right) r+i}$ for $1 \leq i \leq r_{1}-1$.

This completes the proof of Lemma 3.9.

Let $\psi_{q_{1} r+i}=\beta_{\left(q_{1}-1\right) r+r_{1}+i}$ for $i=0, \cdots, r_{1}-1$. We have

THEOREM 3.10 .

$$
\Delta^{r_{1}}(\overbrace{\Delta^{r} \cdots \Delta^{r}}^{q_{1}} I\left(\mu_{n, r}\right))=I\left(\mu_{n, r}\right)+\left(\psi_{0}, \cdots, \psi_{q_{1} r-1}, \psi_{q_{1} r},\right.
$$
$\left.\cdots, \psi_{q_{1} r+r_{1}-1}\right)$.

Proof. By Lemma 3.5, Theorem 3.6 and Proposition 3.8, we only need to prove that any $\left(r-r_{1}+1\right) \times\left(r-r_{1}+1\right)$ minor of $\left(\frac{\partial \psi_{\left(q_{1}-1\right) r+i}}{\partial b_{j}}\right)$ can be generated by $\psi_{0}, \cdots, \psi_{q_{1} r-1}$ and $\psi_{q_{1} r}, \cdots, \psi_{q_{1} r+r_{1}-1}$.

It is easy to deduce that $\left(\frac{\partial \psi_{\left(q_{1}-1\right) r+i}}{\partial b_{j}}\right)=\left(\frac{\partial^{q_{1}} d_{i}}{\partial^{q_{1}-1} b_{0} \partial b_{j}}\right)=$ $\left(\begin{array}{ll}0 & I_{r}\end{array}\right)(-1)^{q_{1}} q_{1} ! B^{-q_{1}}\left(\frac{\partial B}{\partial b_{0}}\right)^{q_{1}-1} D=\left(\begin{array}{ll}0 & I_{r}\end{array}\right)(-1)^{q_{1}} q_{1} ! B^{-q_{1}} L_{n}^{\left(q_{1}-1\right) r} \widehat{D}\left(\begin{array}{c}I_{r} \\ 0\end{array}\right) \propto$ $\left(\begin{array}{ll}0 & I_{r}\end{array}\right) B^{-q_{1}} L_{n}^{\left(q_{1}-1\right) r} \widehat{D}\left(\begin{array}{c}I_{r} \\ 0\end{array}\right)$. So we only need to prove that any $\left(r-r_{1}+1\right) \times\left(r-r_{1}+1\right)$ minor of $\left(\begin{array}{ll}0 & I_{r}\end{array}\right) B^{-q_{1}} L_{n}^{\left(q_{1}-1\right) r} \widehat{D}\left(\begin{array}{c}I_{r} \\ 0\end{array}\right)$, and hence $\left(I_{r}+\alpha_{n-1} L_{r}+\cdots+\alpha_{n-r+1} L_{r}^{r-1}\right)^{-1}\left(0, I_{r}\right) L_{n}^{\left(q_{1}-1\right) r} B^{-q_{1}} \widehat{D}\left(\begin{array}{c}I_{r} \\ 0\end{array}\right)=\left(\begin{array}{cc}* & I_{r-r_{1}} \\ K & 0\end{array}\right)$, can be generated by $\psi_{0}, \cdots, \psi_{q_{1}-1}$ and $\psi_{q_{1} r}, \cdots, \psi_{q_{1} r+r_{1}-1}$. Any $\left(r-r_{1}+1\right) \times\left(r-r_{1}+1\right)$ minor of $\left(\begin{array}{cc}* & I_{r-r_{1}} \\ K & 0\end{array}\right)$ must contain a row with elements either in $K$ or equal to zero. Expanding this minor along that row gives that elements in $K$ generate the minor. By the proof of Lemma 3.9, each element in $K$ can be generated by $\psi_{0}, \cdots, \psi_{q_{1} r-1}$ and $\beta_{\left(q_{1}-1\right) r+r_{1}}, \cdots, \beta_{\left(q_{1}-1\right) r+2 r_{1}-1}$. By the definition of $\left(\psi_{q_{1} r}, \cdots, \psi_{q_{1} r+r_{1}-1}\right)$, we have that $\psi_{0}, \cdots, \psi_{q_{1} r-1}$ and $\psi_{q_{1} r}, \cdots, \psi_{q_{1} r+r_{1}-1}$ generate all $\left(r-r_{1}+1\right) \times\left(r-r_{1}+1\right)$ minors of $\left(\frac{\partial \psi_{\left(q_{1}-1\right) r+i}}{\partial b_{j}}\right)$. This completes the proof of Theorem 3.10.

Denote $f_{0}(x)=f(x), f_{1}(x)=g(x), h_{0}(x)=h(x), r_{-1}=n$ and $r_{0}=r$. We will show that a sequence of monic polynomials $f_{0}(x), f_{1}(x), f_{2}(x), \cdots, f_{k+1}(x)$ can be produced inductively starting from $f_{0}(x)$ and $f_{1}(x)$ such that the degree of $f_{i}(x)$ is $r_{i-1}$ and each product of $h_{i}(x)=f_{i}(x) f_{i+1}(x)$ gives a map $\mu_{r_{i-1}, r_{i}}: \mathbb{C}^{r_{i-1}} \times \mathbb{C}^{r_{i}} \rightarrow \mathbb{C}^{r_{i-1}+r_{i}}$ with the property that the polynomials generated at each of the first $\left(q_{i+1}+1\right)$ steps of the critical extensions of $I\left(\mu_{r_{i-1}, r_{i}}\right)$ can be added into the corresponding steps to form the critical extensions of $I\left(\mu_{n, r}\right)$.

Recall that $B^{-q_{1}} \widehat{A}=I_{n}+\gamma_{n-1} L_{n}+\cdots+\gamma_{1} L_{n}^{n-1}$. Let $f_{2}(x)=x^{r_{1}}+\gamma_{n-1} x^{r_{1}-1}+$ $\cdots+\gamma_{n-r_{1}}$. Then the product $h_{1}(x)=f_{1}(x) f_{2}(x)=g(x) f_{2}(x)=x^{n}+\sigma_{r+r_{1}-1} x^{n-1}+$ $\cdots+\sigma_{0}$ gives a map $\mu_{r, r_{1}}: \mathbb{C}^{r} \times \mathbb{C}^{r_{1}} \rightarrow \mathbb{C}^{r+r_{1}}$. Taking derivatives of the coefficients of $h_{1}(x)$ with respect to $b_{r-1}, \cdots, b_{0}, \gamma_{n-1}, \cdots, \gamma_{n-r_{1}}$ gives its first Jacobian

$$
\delta I\left(\mu_{r, r_{1}}\right)=\left(\left(I_{r+r_{1}}, 0\right) \Gamma_{r+r_{1}+1}\left(\begin{array}{c}
I_{r} \\
0
\end{array}\right),\left(I_{r+r_{1}}, 0\right) B_{r+r_{1}+1}\left(\begin{array}{c}
I_{r_{1}} \\
0
\end{array}\right)\right)
$$

where $\Gamma_{r+r_{1}+1}=I_{r+r_{1}+1}+\gamma_{n-1} L_{r+r_{1}+1}+\cdots+\gamma_{n-r_{1}} L_{r+r_{1}+1}^{r_{1}}$ and $B_{r+r_{1}+1}=I_{r+r_{1}+1}+$ $b_{r-1} L_{r+r_{1}+1}+\cdots+b_{0} L_{r+r_{1}+1}^{r}$.

Repeating the same process as we did for $I\left(\mu_{n, r}\right)$, we get polynomials $\varphi_{0}, \cdots, \varphi_{r_{1}-1}, \cdots, \varphi_{\left(q_{2}-1\right) r_{1}}, \cdots, \varphi_{q_{2} r_{1}-1}$ and $\varphi_{q_{2} r_{1}}, \cdots, \varphi_{q_{2} r_{1}+r_{2}-1}$ which satisfy 


$$
\begin{gathered}
\left(\frac{\partial \varphi_{s r_{1}+i}}{\partial \gamma_{n-j}}\right)=-(s+1)\left(\frac{\partial \varphi_{s r_{1}+i}}{\partial b_{j}}\right)\left(I_{r}, 0\right) \Gamma_{r+r_{1}}^{-1}\left(I_{r+r_{1}}, 0\right) B_{r+r_{1}+1}\left(\begin{array}{c}
I_{r_{1}} \\
0
\end{array}\right) \text { for } s=0, \cdots, \\
q_{2}-1, i=0, \cdots, r_{1}-1 \text { and } j=1, \cdots, r_{1} \text {, where } \Gamma_{r+r_{1}}=\left(I_{r+r_{1}}, 0\right) \Gamma_{r+r_{1}+1}\left(\begin{array}{c}
I_{r+r_{1}} \\
0
\end{array}\right)
\end{gathered}
$$

and

$$
\begin{array}{r}
\overbrace{\Delta^{r_{1}} \cdots \Delta^{r_{1}}}^{s} I\left(\mu_{r, r_{1}}\right)=I\left(\mu_{r, r_{1}}\right)+\left(\varphi_{0}, \cdots, \varphi_{s r_{1}-1}\right) \text { for } s=1, \cdots, q_{2} \\
\Delta^{r_{2}}(\overbrace{\Delta^{r_{1}} \cdots \Delta^{r_{1}}}^{q_{2}} I\left(\mu_{r, r_{1}}\right))=I\left(\mu_{r, r_{1}}\right)+\left(\varphi_{0}, \cdots, \varphi_{q_{2} r_{1}-1}, \varphi_{q_{2} r_{1}}, \cdots, \varphi_{q_{2} r_{1}+r_{2}-1}\right) .
\end{array}
$$

We will prove that adding these polynomials correspondingly into the generator sets gives the critical extensions of $\overbrace{\Delta^{r} \cdots \Delta^{r}}^{q_{1}} I\left(\mu_{n, r}\right)$.

The following lemma is true.

Lemma 3.11. For any $s\left(1 \leq s \leq q_{1}\right)$, the coefficients of $L_{n+r+1}^{i}$ in $B_{n+r+1}^{-s} A_{n+r+1}$ for $i=n-s r+1, \cdots, n+r$ are zeros $\bmod \left(\psi_{0}, \cdots, \psi_{s r-1}\right)$.

Proof. The case $s=1$ was proved at the beginning of this subsection.

Suppose that we proved Lemma 3.11 for $s \leq p$. If $p=q_{1}$, we are done. Otherwise, we may assume that $1 \leq p<q_{1}$. We will show that the coefficients of $L_{n+r+1}^{i}$ in $B_{n+r+1}^{-(p+1)} A_{n+r+1}$ for $i=n-(p+1) r+1, \cdots, n+r$ are zeros $\bmod \left(\psi_{0}, \cdots, \psi_{(p+1) r-1}\right)$.

Denote $B_{n+r+1}^{-(p+1)} A_{n+r+1}=I_{n+r+1}+\lambda_{n-1} L_{n+r+1}+\cdots+\lambda_{0} L_{n+r+1}^{n}+\lambda_{-1} L_{n+r+1}^{n+1}+$ $\cdots+\lambda_{-r} L_{n+r+1}^{n+r}$ and $B_{n+r+1}^{-p} A_{n+r+1}=I_{n+r+1}+\kappa_{n-1} L_{n+r+1}+\cdots+\kappa_{0} L_{n+r+1}^{n}+$ $\kappa_{-1} L_{n+r+1}^{n+1}+\cdots+\kappa_{-r} L_{n+r+1}^{n+r}$. By inductive assumption $\kappa_{n-i} \equiv 0 \bmod \left(\psi_{0}, \cdots, \psi_{p r-1}\right)$ for $i=n-p r+1, \cdots, n+r$.

By Equation (3.7), we have $\left(I_{n}, 0\right)(-1)^{p} p ! B_{n+r+1}^{-(p+1)} L_{n+r+1}^{(p-1) r} A_{n+r+1}\left(\begin{array}{c}I_{r} \\ 0\end{array}\right)=$ $(-1)^{p} p ! B^{-p-1} L_{n}^{(p-1) r} A=\left(\frac{\partial^{p} d_{i}}{\partial^{p-1} b_{0} \partial b_{j}}\right)$. Left multiplying this equation by $e_{n-r+1}$ gives that $\lambda_{p r+i} \propto \frac{\partial^{p} d_{r-1}}{\partial^{p-1} b_{0} \partial b_{r-1-i}}=\psi_{p r+i}$ for $i=0, \cdots, r-1$, where $e_{n-r+1}$ is a $1 \times n$ vector with 1 in the $(n-r+1)$ position and zero elsewhere. Comparing the coefficients of $L_{n+r+1}^{i}$ in both sides of the equation $\left(B_{n+r+1}^{-(p+1)} A_{n+r+1}\right) B_{n+r+1}=B_{n+r+1}^{-p} A_{n+r+1}$ for $i=n-p r+1, \cdots, n+r$, we have that

$$
\begin{aligned}
& \lambda_{n-i}+\lambda_{n-i+1} b_{r-1}+\cdots+\lambda_{n-i+r} b_{0} \equiv 0 \bmod \left(\psi_{0}, \cdots, \psi_{(p+1) r-1}\right) \\
& \text { for } i=n-p r+1, \cdots, n+r
\end{aligned}
$$

Using Equation (3.22) and $\lambda_{p r+i} \propto \psi_{p r+i}$ for $i=0, \cdots, r-1$, we immediately have that $\lambda_{n-i} \equiv 0 \bmod \left(\psi_{0}, \cdots, \psi_{(p+1) r-1}\right)$ for $i=n-p r+1, \cdots, n+r$. Because $\lambda_{p r+i}=\lambda_{n-(n-p r-i)}$ for $i=0, \cdots, r-1$, so $\lambda_{n-i} \equiv 0 \bmod \left(\psi_{0}, \cdots, \psi_{(p+1) r-1}\right)$ for $i=$ $n-(p+1) r+1, \cdots, n-p r$ as well. This completes the proof of Lemma 3.11.

From Lemma 3.11, we have the following proposition. 
Proposition 3.12. $\varphi_{i} \equiv \psi_{q_{1} r+i} \bmod \left(\psi_{0}, \cdots, \psi_{q_{1} r-1}\right)$ for $i=0, \cdots, r_{1}-1$.

Proof. From the first Jacobian $\delta I\left(\mu_{r, r_{1}}\right)$, we have that $\Gamma_{r+r_{1}}^{-1} \delta I\left(\mu_{r, r_{1}}\right)=$ $\left(\left(\begin{array}{c}I_{r} \\ 0\end{array}\right), W\left(\begin{array}{c}I_{r_{1}} \\ 0\end{array}\right)\right)$, where $W=\Gamma_{r+r_{1}}^{-1}\left(I_{r+r_{1}}, 0\right) B_{r+r_{1}+1}\left(\begin{array}{c}I_{r+r_{1}} \\ 0\end{array}\right)$. Denote $W$ as $I_{r+r_{1}}+w_{r-1} L_{r+r_{1}}+\cdots+w_{0} L_{r+r_{1}}^{r}+w_{-1} L_{r+r_{1}}^{r+1}+\cdots+w_{-r_{1}+1} L_{r+r_{1}}^{r+r_{1}-1}$. We have that $w_{i}=\varphi_{i}$ for $i=0, \cdots, r_{1}-1$.

By Lemma 3.11, $\left(I_{r+r_{1}}, 0\right) B_{n+r+1}^{-q_{1}} A_{n+r+1}\left(\begin{array}{c}I_{r+r_{1}} \\ 0\end{array}\right) \equiv I_{r+r_{1}}+\gamma_{n-1} L_{r+r_{1}}+\cdots+$ $\gamma_{n-r_{1}} L_{r+r_{1}}^{r_{1}} \equiv I_{r+r_{1}}+\gamma_{n-1} L_{r+r_{1}}+\cdots+\gamma_{n-r} L_{r+r_{1}}^{r} \bmod \left(\psi_{0}, \cdots, \psi_{q_{1} r-1}\right)$. Comparing the coefficients of $L_{n}^{k}$ in both sides of Equation (3.17) and $L_{r+r_{1}}^{k}$ in both sides of $\left(I_{r+r_{1}}+\gamma_{n-1} L_{r+r_{1}}+\cdots+\gamma_{n-r} L_{r+r_{1}}^{r}\right)\left(I_{r+r_{1}}+w_{r-1} L_{r+r_{1}}+\cdots+w_{0} L_{r+r_{1}}^{r}+w_{-1} L_{r+r_{1}}^{r+1}+\right.$ $\left.\cdots+w_{-r_{1}+1} L_{r+r_{1}}^{r+r_{1}-1}\right) \equiv \Gamma_{r+r_{1}} W=\left(I_{r+r_{1}}, 0\right) B_{r+r_{1}+1}\left(\begin{array}{c}I_{r+r_{1}} \\ 0\end{array}\right)=I_{r+r_{1}}+b_{r-1} L_{r+r_{1}}+$ $\cdots+b_{0} L_{r+r_{1}}^{r} \bmod \left(\psi_{0}, \cdots, \psi_{q_{1} r-1}\right)$ for $k=1, \cdots, r$, we have that

$$
\begin{array}{r}
\beta_{n-k}+\beta_{n-k+1} \gamma_{n-1}+\cdots+\beta_{n-1} \gamma_{n-k+1}+\gamma_{n-k}=b_{r-k} \equiv w_{r-k}+w_{r-k+1} \gamma_{n-1}+ \\
\cdots+w_{r-1} \gamma_{n-k+1}+\gamma_{n-k} \bmod \left(\psi_{0}, \cdots, \psi_{q_{1} r-1}\right) \text { for } k=1, \cdots, r
\end{array}
$$

Let $k=1$ in Equation (3.23), we have $\beta_{n-1}+\gamma_{n-1} \equiv w_{r-1}+\gamma_{n-1}$, so $\beta_{n-1} \equiv w_{r-1}$. Let $k=2, \cdots, r$ in Equation (3.23) and use induction, we have $\beta_{n-k} \equiv w_{r-k}$ for each $k=1, \cdots, r$, so $\varphi_{i}=w_{i} \equiv \beta_{n-r+i}=\beta_{\left(q_{1}-1\right) r+r_{1}+i}=\psi_{q_{1} r+i} \bmod \left(\psi_{0}, \cdots, \psi_{q_{1} r-1}\right)$ for $i=0, \cdots, r_{1}-1$.

As an immediate corollary of Theorem 3.10 and Proposition 3.12, we have

Corollary 3.13. $\Delta^{r_{1}}(\overbrace{\Delta^{r} \cdots \Delta^{r}}^{q_{1}} I\left(\mu_{n, r}\right))=I\left(\mu_{n, r}\right)+\left(\psi_{0}, \cdots, \psi_{q_{1} r-1}, \varphi_{0}\right.$, $\left.\cdots, \varphi_{r_{1}-1}\right)$.

Now we can prove the following theorem.

THEOREM 3.14. $\overbrace{\Delta^{r_{1}} \cdots \Delta^{r_{1}} \Delta^{r} \cdots \Delta^{r}}^{s} I\left(\mu_{n, r}\right)=I\left(\mu_{n, r}\right)+\left(\psi_{0}, \cdots, \psi_{q_{1} r-1}, \varphi_{0}\right.$, $\left.\cdots, \varphi_{s r_{1}-1}\right)$ for $s=1, \cdots, q_{2}$ and $\Delta^{r_{2}}(\overbrace{\Delta^{r_{1}} \cdots \Delta^{r_{1}} \Delta^{r} \cdots \Delta^{r}}^{q_{2}} I\left(\mu_{n, r}\right)=I\left(\mu_{n, r}\right)+$ $\left(\psi_{0}, \cdots, \psi_{q_{1} r-1}, \varphi_{0}, \cdots, \varphi_{q_{2} r_{1}-1}, \varphi_{q_{2} r_{1}}, \cdots, \varphi_{q_{2} r_{1}+r_{2}-1}\right)$.

We need the following two lemmas.

LEMMA 3.15. $\left(\psi_{0}, \cdots, \psi_{q_{1}-1}, \gamma_{n-r_{1}}, \cdots, \gamma_{n-1}, b_{0}, \cdots, b_{r-1}\right)$ forms a new local coordinate system around zero on $\mathbb{C}^{n} \times \mathbb{C}^{r}$.

Proof. Let $A_{n+1}=\left(I_{n+1}, 0\right) A_{n+r+1}\left(\begin{array}{c}I_{n+1} \\ 0\end{array}\right)$ and $B_{n+1}=$ $\left(I_{n+1}, 0\right) B_{n+r+1}\left(\begin{array}{c}I_{n+1} \\ 0\end{array}\right)$. Then we have that $A_{n+1}=I_{n+1}+a_{n-1} L_{n+1}+\cdots+a_{0} L_{n+1}^{n}$, $B_{n+1}=I_{n+1}+b_{r-1} L_{n+1}+\cdots+b_{0} L_{n+1}^{r}$ and $B_{n+1}^{-q_{1}} A_{n+1}=I_{n+1}+\gamma_{n-1} L_{n+1}+\cdots+$ $\gamma_{0} L_{n+1}^{n}$. By Lemma 3.1(2) and the equation $A_{n+1}=\left(B_{n+1}^{-q_{1}} A_{n+1}\right) B_{n+1}^{q_{1}}$, it is easy to see that $\gamma_{0}, \cdots, \gamma_{n-1}, b_{0}, \cdots, b_{r-1}$ form a new local coordinate system around zero on $\mathbb{C}^{n} \times \mathbb{C}^{r}$. 
By Lemma 3.11, $\gamma_{i} \equiv 0 \bmod \left(\psi_{0}, \cdots, \psi_{q_{1} r-1}\right)$ for $i=0, \cdots, q_{1} r-1$. So to prove Lemma 3.15 , it is sufficient to prove that $\psi_{0}, \cdots, \psi_{q_{1} r-1}$ are polynomials in $\gamma_{0}, \cdots, \gamma_{n-1}, b_{0}, \cdots, b_{r-1}$. This follows directly from the fact that $\psi_{i}$ s are polynomials in $a_{0}, \cdots, a_{n-1}, b_{0}, \cdots, b_{r-1}$ while $a_{0}, \cdots, a_{n-1}, b_{0}, \cdots, b_{r-1}$ are polynomials in $\gamma_{0}, \cdots, \gamma_{n-1}, b_{0}, \cdots, b_{r-1}$.

Denote $h_{1}(x) g(x)^{q_{1}}=x^{n+r}+\tau_{n+r-1} x^{n+r-1}+\cdots+\tau_{0}$. We have

LEMma 3.16. $c_{i} \equiv \tau_{i} \bmod \left(\psi_{0}, \cdots, \psi_{q_{1} r-1}\right)$ for $i=0, \cdots, n+r-1$.

Proof. Let $C_{n+r+1}=I_{n+r+1}+c_{n+r-1} L_{n+r+1}+\cdots+c_{0} L_{n+r+1}^{n+r}$, where $c_{i} \mathrm{~s}$ are the coefficients of $h(x)$. Then $C_{n+r+1}=A_{n+r+1} B_{n+r+1}$. By Lemma 3.11, $B_{n+r+1}^{-q_{1}} A_{n+r+1} \equiv I_{n+r+1}+\gamma_{n-1} L_{n+r+1}+\cdots+\gamma_{n-r_{1}} L_{n+r+1}^{r_{1}} \bmod \left(\psi_{0}, \cdots, \psi_{q_{1} r-1}\right)$. So $C_{n+r+1}=A_{n+r+1} B_{n+r+1}=\left(B_{n+r+1}^{-q_{1}} A_{n+r+1}\right) B_{n+r+1}^{q_{1}+1} \equiv\left(I_{n+r+1}+\right.$ $\left.\gamma_{n-1} L_{n+r+1}+\cdots+\gamma_{n-r_{1}} L_{n+r+1}^{r_{1}}\right) B_{n+r+1}^{q_{1}+1}=I_{n+r+1}+\tau_{n+r-1} L_{n+r+1}+\cdots+$ $\tau_{0} L_{n+r+1}^{n+r} \bmod \left(\psi_{0}, \cdots, \psi_{q_{1} r-1}\right)$. Therefore $c_{i} \equiv \tau_{i} \bmod \left(\psi_{0}, \cdots, \psi_{q_{1} r-1}\right)$ for $i=$ $0, \cdots, n+r-1$. $\square$

Proof of Theorem 3.14. The case $s=1$ is Corollary 3.13.

Suppose we have proved that

$\overbrace{\Delta^{r_{1}} \cdots \Delta^{r_{1}} \Delta^{r} \cdots \Delta^{r}}^{q_{1}} I\left(\mu_{n, r}\right)=I\left(\mu_{n, r}\right)+\left(\psi_{0}, \cdots, \psi_{q_{1} r-1}, \varphi_{0}, \cdots, \varphi_{s r_{1}-1}\right)$ for $s=$ $1, \cdots, p$.

If $p=q_{2}$, we have done the first part of Theorem 3.14. Otherwise, we may assume $p<q_{2}$. We will show that

$\overbrace{\Delta^{r_{1}} \cdots \Delta^{r_{1}} \Delta^{r} \cdots \Delta^{r}}^{p+1} I\left(\mu_{n, r}\right)=I\left(\mu_{n, r}\right)+\left(\psi_{0}, \cdots, \psi_{q_{1} r-1}, \varphi_{0}, \cdots, \varphi_{(p+1) r_{1}-1}\right)$.

By Lemma 3.16, $\overbrace{\Delta^{r_{1}} \cdots \Delta^{r_{1}} \Delta^{r} \cdots \Delta^{r}}^{q_{1}} I\left(\mu_{n, r}\right)=I\left(\mu_{n, r}\right)+\left(\psi_{0}, \cdots, \psi_{q_{1} r-1}, \varphi_{0}\right.$, $\left.\cdots, \varphi_{p r_{1}-1}\right)=\left(c_{n+r-1}, \cdots, c_{0}, \psi_{0}, \cdots, \psi_{q_{1} r-1}, \varphi_{0}, \cdots, \varphi_{p r_{1}-1}\right)=\left(\tau_{n+r-1}, \cdots, \tau_{0}\right.$, $\left.\psi_{0}, \cdots, \psi_{q_{1} r-1}, \varphi_{0}, \cdots, \varphi_{p r_{1}-1}\right) . \quad$ Considering $\delta \overbrace{\Delta^{r_{1}} \cdots \Delta^{r_{1}} \Delta^{r} \cdots \Delta^{r}}^{q_{1}} I\left(\mu_{n, r}\right) \quad=$ $\delta\left(\tau_{n+r-1}, \cdots, \tau_{0}, \psi_{0}, \cdots, \psi_{q_{1} r-1}, \varphi_{0}, \cdots, \varphi_{p r_{1}-1}\right)$ with derivatives taken with respect to the new coordinate system in the order $\gamma_{n-1}, \cdots, \gamma_{n-r_{1}}, \psi_{q_{1} r-1}, \cdots, \psi_{0}, b_{r-1}$, $\cdots, b_{0}$, we have that it is given by the following matrix

$$
\left(\begin{array}{ccc}
B_{n+r}^{q_{1}+1}\left(\begin{array}{c}
I_{r_{1}} \\
0
\end{array}\right) & 0 & \left(q_{1}+1\right) B_{n+r}^{q_{1}} \Gamma_{n+r}\left(\begin{array}{c}
I_{r} \\
0
\end{array}\right) \\
0 & I_{q_{1} r} & 0 \\
\left(\frac{\partial \varphi_{s r_{1}+i}}{\partial \gamma_{n-j}}\right) & 0 & \left(\frac{\partial \varphi_{s r_{1}+i}}{\partial b_{j}}\right)
\end{array}\right)
$$

where $\Gamma_{n+r}=I_{n+r}+\gamma_{n-1} L_{n+r}+\cdots+\gamma_{n-r_{1}} L_{n+r}^{r_{1}}$ and $s$ varies from 0 to $p-1$.

Left multiplying matrix (3.24) by $\left(\begin{array}{cc}B_{n+r}^{-q_{1}} & 0 \\ 0 & I_{q_{1} r+p r_{1}}\end{array}\right)$, we have 


$$
\begin{aligned}
& \left(\begin{array}{ccc}
B_{n+r}\left(\begin{array}{c}
I_{r_{1}} \\
0
\end{array}\right) & 0 & \left(q_{1}+1\right) \Gamma_{n+r}\left(\begin{array}{c}
I_{r} \\
0
\end{array}\right) \\
0 & I_{q_{1} r} & 0 \\
\left(\frac{\partial \varphi_{s r_{1}+i}}{\partial \gamma_{n-j}}\right) & 0 & \left(\frac{\partial \varphi_{s r_{1}+i}}{\partial b_{j}}\right)
\end{array}\right) \\
& =\left(\begin{array}{ccc}
\left(I_{r+r_{1}}, 0\right) B_{r+r_{1}+1}\left(\begin{array}{c}
I_{r_{1}} \\
0
\end{array}\right) & 0 & \left(q_{1}+1\right) \Gamma_{r+r_{1}}\left(\begin{array}{c}
I_{r} \\
0
\end{array}\right) \\
0 & 0 & 0 \\
0 & I_{q_{1} r} & 0 \\
\left(\frac{\partial \varphi_{s r_{1}+i}}{\partial \gamma_{n-j}}\right) & 0 & \left(\frac{\partial \varphi_{s r_{1}+i}}{\partial b_{j}}\right)
\end{array}\right) .
\end{aligned}
$$

Left multiplying (3.25) by

$$
\left(\begin{array}{cc}
\frac{1}{q_{1}+1} \Gamma_{r+r_{1}}^{-1} & 0 \\
0 & I_{2 q_{1} r+p r_{1}}
\end{array}\right)\left(\begin{array}{ccc}
I_{r+r_{1}} & 0 & 0 \\
0 & I_{2 q_{1} r} & 0 \\
\frac{-1}{q_{1}+1}\left(\frac{\partial \varphi_{s r_{1}+i}}{\partial b_{j}}\right)\left(I_{r}, 0\right) \Gamma_{r+r_{1}}^{-1} & 0 & I_{p r_{1}}
\end{array}\right)
$$

and using Equation (3.20), we have that

$$
\left(\begin{array}{ccc}
\frac{1}{q_{1}+1} \Gamma_{r+r_{1}}^{-1}\left(I_{r+r_{1}}, 0\right) B\left(\begin{array}{c}
I_{r_{1}} \\
0
\end{array}\right) & 0 & \left(\begin{array}{c}
I_{r} \\
0
\end{array}\right) \\
0 & 0 & 0 \\
0 & I_{q_{1} r} & 0 \\
\left(1+\frac{1}{(s+1)\left(q_{1}+1\right)}\right)\left(\frac{\partial \varphi_{s r_{1}+i}}{\partial \gamma_{n-j}}\right) & 0 & 0
\end{array}\right)
$$

By induction and the construction of $\varphi_{i} \mathrm{~s}$, the matrix (3.26) is equal to

$$
\left(\begin{array}{cccc}
\frac{1}{q_{1}+1}\left(I_{r}, 0\right) \Gamma_{r+r_{1}}^{-1}\left(I_{r+r_{1}}, 0\right) B\left(\begin{array}{c}
I_{r_{1}} \\
0
\end{array}\right) & 0 & I_{r} \\
0 & 0 & 0 \\
0 & I_{q_{1} r} & 0 \\
0 & 0 & 0
\end{array}\right) \bmod \left(\varphi_{0}, \cdots, \varphi_{(p+1) r_{1}-1}\right)
$$

So the corank of $\delta \overbrace{\Delta^{r_{1}} \cdots \Delta^{r_{1}} \Delta^{r} \cdots \Delta^{r}}^{p} I\left(\mu_{n, r}\right)$ is $r_{1}$ and any $\left(n+r-r_{1}+1\right)$ minor is generated by $\left(\varphi_{0}, \cdots, \varphi_{(p+1) r_{1}-1}\right)$. Because each $\varphi_{i}$ is only different from a $\left(n+r-r_{1}+1\right)$ minor of $\delta \overbrace{\Delta^{r_{1}} \cdots \Delta^{r_{1}} \Delta^{r} \cdots \Delta^{r}}^{p} I\left(\mu_{n, r}\right)$ by a nonzero constant, so

$$
\overbrace{\Delta^{r_{1}} \cdots \Delta^{r_{1}} \Delta^{r} \cdots \Delta^{r}}^{p+1} I\left(\mu_{n, r}\right)=I\left(\mu_{n, r}\right)+\left(\psi_{0}, \cdots, \psi_{q_{1} r-1}, \varphi_{0}, \cdots, \varphi_{(p+1) r_{1}-1}\right) .
$$

This completes the proof of the first part of Theorem 3.14. to

Using the same idea, we can prove that $\delta \overbrace{\Delta^{r_{1}} \cdots \Delta^{r_{1}} \Delta^{r} \cdots \Delta^{r}}^{q_{2}} I\left(\mu_{n, r}\right)$ is equivalent 


$$
\left(\begin{array}{ccc}
\frac{1}{q_{1}+1}\left(I_{r}, 0\right) \Gamma_{r+r_{1}}^{-1}\left(I_{r+r_{1}}, 0\right) B\left(\begin{array}{c}
I_{r_{1}} \\
0
\end{array}\right) & 0 & I_{r} \\
0 & 0 & 0 \\
0 & I_{q_{1} r} & 0 \\
0 & 0 & 0 \\
\left(1+\frac{1}{q_{2}\left(q_{1}+1\right)}\right)\left(\frac{\partial \varphi\left(q_{2}-1\right) r_{1}+i}{\partial \gamma_{n-j}}\right) & 0 & 0
\end{array}\right) \bmod \left(\varphi_{0}, \cdots, \varphi_{q_{2} r_{1}-1}\right)
$$

By comparison with $\delta \overbrace{\Delta^{r_{1}} \cdots \Delta^{r_{1}}}^{q_{2}} I\left(\mu_{r, r_{1}}\right)$ which is equivalent to

$$
\left(\begin{array}{cc}
\frac{1}{q_{1}+1}\left(I_{r}, 0\right) \Gamma_{r+r_{1}}^{-1}\left(I_{r+r_{1}}, 0\right) B\left(\begin{array}{c}
I_{r_{1}} \\
0
\end{array}\right) & I_{r} \\
0 & 0 \\
\left(1+\frac{1}{q_{2}}\right)\left(\frac{\partial \varphi_{\left(q_{2}-1\right) r_{1}+i}}{\partial \gamma_{n-j}}\right) & 0
\end{array}\right) \bmod \left(\varphi_{0}, \cdots, \varphi_{q_{2} r_{1}-1}\right)
$$

the matrices (3.28) and (3.29) have the same corank. By induction, the latter one has corank $r_{2}$, so does the matrix (3.28). All the $\left(n+r-r_{2}+1\right)$ minors of matrix (3.28) and the $\left(r+r_{1}-r_{2}+1\right)$ minors of matrix (3.29) generate the same idea, so

$$
\begin{aligned}
& \Delta^{r_{2}} \overbrace{\Delta^{r_{1}} \cdots \Delta^{r_{1}} \Delta^{r} \cdots \Delta^{r}}^{q_{2}} I\left(\mu_{n, r}\right)=I\left(\mu_{n, r}\right)+\left(\psi_{0}, \cdots, \psi_{q_{1} r-1}, \varphi_{0}, \cdots, \varphi_{q_{2} r_{1}-1},\right. \\
& \left.\varphi_{q_{2} r_{1}}, \cdots, \varphi_{q_{2} r_{1}+r_{2}-1}\right) . \square
\end{aligned}
$$

As a consequence of Theorem 3.14, we have that

COROllary 3.17. The first $\left(q_{1}+q_{2}+1\right)$ entries in $T B\left(I\left(\mu_{n, r}\right)\right)$ is $\left(r, \cdots, r, r_{1}, \cdots, r_{1}, r_{2}\right)$ with $r$ repeating $q_{1}$ times and $r_{1}$ repeating $q_{2}$ times.

Replacing $f_{0}(x), f_{1}(x)$ with $f_{1}(x), f_{2}(x)$ and repeating the same process, we can produce $f_{3}(x)$ of degree $r_{2}$ and a map $\mu_{r_{1}, r_{2}}: \mathbb{C}^{r_{1}} \times \mathbb{C}^{r_{2}} \rightarrow \mathbb{C}^{r_{1}+r_{2}}$. Using the first $\left(q_{3}+1\right)$ critical extensions of $I\left(\mu_{r_{1}, r_{2}}\right)$, we can generate polynomials $\varphi_{q_{2} r_{1}}, \cdots, \varphi_{q_{2} r_{1}+q_{3} r_{2}-1}, \varphi_{q_{2} r_{1}+q_{3} r_{2}}, \cdots, \varphi_{q_{2} r_{1}+q_{3} r_{2}+r_{3}-1}$ such that they can be added correspondingly into the generator set to form the next $\left(q_{3}+1\right)$ critical extensions of $I\left(\mu_{n, r}\right)$. Repeating the same procedure over and over, we can produce $f_{4}(x), \cdots, f_{k+1}(x)$ and use them to prove $T B\left(I\left(\mu_{n, r}\right)\right)=I(n, r)$. Due to the heavy notations, we will not do so here. Instead, we mention a key observation that explains why we can add polynomials at each step to obtain the corresponding critical extension of $I\left(\mu_{n, r}\right)$. At the $\left(q_{1}+\cdots+q_{p}+s\right)$-th step of the critical extension of $I\left(\mu_{n, r}\right)$ for some $1 \leq s \leq q_{p+1}$, the Jacobian matrix is equivalent to a matrix with the form

$$
\left(\begin{array}{ccc}
I_{r_{p-1}} & 0 & \mu \Lambda \\
0 & 0 & 0 \\
0 & I_{q_{1} r+q_{2} r_{1}+\cdots+q_{p} r_{p-1}} & 0 \\
0 & 0 & 0 \\
0 & 0 & \nu \Theta
\end{array}\right) \bmod \left(\varphi_{q_{2}+\cdots+q_{p}}, \cdots, \varphi_{q_{2}+\cdots+q_{p}+s r_{p}-1}\right)
$$

for some nonzero constant $\mu$ and $\nu$.

$$
\text { The matrix } \delta \overbrace{\Delta^{r_{p-1}} \cdots \Delta^{r_{p-1}}}^{s} I\left(\mu_{r_{p-1}, r_{p}}\right) \text { is equivalent to }
$$




$$
\left(\begin{array}{cc}
I_{r_{p-1}} & \Lambda \\
0 & 0 \\
0 & \nu^{\prime} \Theta
\end{array}\right) \bmod \left(\varphi_{q_{2}+\cdots+q_{p}}, \cdots, \varphi_{q_{2}+\cdots+q_{p}+s r_{p}-1}\right)
$$

for some nonzero constant $\nu^{\prime}$.

It is obvious that the matrices (3.30) and (3.31) have the same corank and the corresponding minors generate the same ideal. So we can add the polynomials generated by the first $\left(q_{p+1}+1\right)$ critical extensions of $I\left(\mu_{r_{p-1}, r_{p}}\right)$ into the corresponding generator sets to form the critical extensions of $I\left(\mu_{n, r}\right)$. Hence Theorem 1.2 is true. This completes the proof of Varley's Conjecture.

\section{REFERENCES}

[1] M. Adams, C. McCrory, T. Shifrin and R. Varley, Invariants of Gauss Maps of Theta Divisors, Proc. Sympos. Pure Math., Vol 54, part 2, 1-8, Amer. Math. Soc., Providence, RI, 1993.

[2] V. I. Arnol'd, S. M. Guseřn-Zade and A. N. Varchenko, Singularities of Differentiable Maps, Volume I, Monographs in Mathematics, 82, Birhäuser, Boston, 1985.

[3] J. M. Boardman, Singularities of Differential Maps, Inst. Hautes Études Sci. Publ. Math., 33 (1967), pp. 21-57.

[4] J. M ${ }^{c}$ Kernan, Versality for canonical curves and complete intersections, Math. Ann., 308 (1995), pp. 559-569.

[5] R. VARLEY, A Normal Form for the Secant Map Germ, unpublished notes, University of Georgia, 2001.

[6] Y. Wang, J. Lin and M. Ge, A Short Note on the Thom-Boardman Symbols of Differentiable Maps, arXiv:1106.3920.

[7] J. Wethington, On computing the Thom-Boardman symbols for polynomial multiplication maps, Ph.D. Dissertation at the University of Georgia, 2002. 